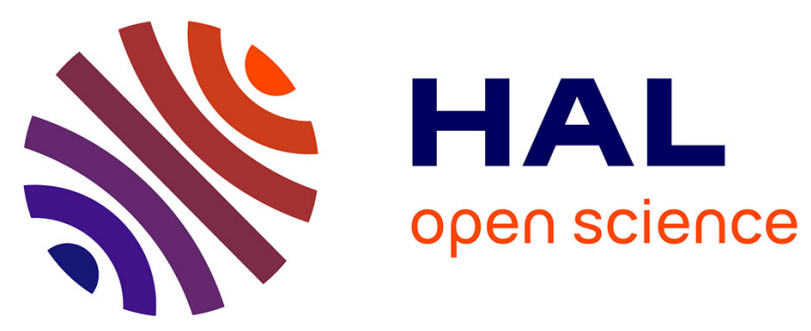

\title{
A new class of multi-dimensional Teager Kaiser and higher order operators based on directional derivatives
}

Fabien Salzenstein, Abdel-Ouahab Boudraa, Thierry Chonavel

\section{To cite this version:}

Fabien Salzenstein, Abdel-Ouahab Boudraa, Thierry Chonavel. A new class of multi-dimensional Teager Kaiser and higher order operators based on directional derivatives. Multidimensional Systems and Signal Processing, 2013, 24 (3), pp.543-572. 10.1007/s11045-012-0179-2 . hal-00704168

\section{HAL Id: hal-00704168 \\ https://hal.science/hal-00704168}

Submitted on 4 Jun 2012

HAL is a multi-disciplinary open access archive for the deposit and dissemination of scientific research documents, whether they are published or not. The documents may come from teaching and research institutions in France or abroad, or from public or private research centers.
L'archive ouverte pluridisciplinaire HAL, est destinée au dépôt et à la diffusion de documents scientifiques de niveau recherche, publiés ou non, émanant des établissements d'enseignement et de recherche français ou étrangers, des laboratoires publics ou privés. 


\title{
A new class of multi-dimensional Teager-Kaiser and higher order operators based on directional derivatives
}

\author{
F. Salzenstein - A. O. Boudraa - T. Chonavel
}

Received: 15 February 2011 / Revised: 17 January 2012 / Accepted: 5 February 2012

C) Springer Science+Business Media, LLC 2012

\begin{abstract}
This work aims at introducing some energy operators linked to Teager-Kaiser energy operator (TKEO) (Kaiser in On a simple algorithm to calculate the energy of a signal, pp 381-384, 1990), its associated higher order versions and expanding them to multidimensional signals. These operators are very useful for analysing oscillatory signals with time-varying amplitude and frequency (AM-FM). We first propose a new mathematical expression of these operators using directional derivatives along any n-D vector and Kronecker powers (Proposition 1, Sect. 3). This mathematical formulation allows us to extend to n-D case some properties of the classical TKEO such as tracking of AM envelope and instantaneous frequency of a multi-dimensional AM-FM signal. In addition, we have introduced a new scalar function using the directional derivative along a vector to recover the "sign" of the frequency components. Applications of this model to a local n-D AM-FM signal and the related demodulation errors are presented. To show the effectiveness and the robustness of the new class of operators in term of envelope and frequency tracking, results obtained on synthetic and real data are compared to multi-dimensional energy separation algorithm (Maragos and Bovik in J Opt Soc Am A 12:1867-1876, 1995) and to our previously developed method (Salzenstein and Boudraa in Signal Process 89(4):623-640, 2009). Finally, the performances of these methods are investigated in the presence of an additive noise.
\end{abstract}

F. Salzenstein $(\varangle)$

Laboratoire INESS, UMR CNRS 7163 Université de Strasbourg, Strasbourg, France

e-mail: salzenst@iness.c-strasbourg.fr

A. O. Boudraa

IRENav, Ecole Navale/Arts et Métiers ParisTech (EA 3634), BCRM Brest, CC 600, 29240 Brest, France e-mail: boudra@ecole-navale.fr

T. Chonavel

Lab-STICC, UMR 3192, TELECOM Bretagne, Technopole Brest Iroise, 29238 Brest, France

e-mail: thierry.chonavel@telecom-bretagne.eu 
Keywords Multi-dimensional Teager-Kaiser energy operator · Multi-dimensional higher order differential operators - Kronecker product - Kronecker power · AM-FM model · Directional derivative $\cdot$ Demodulation

\section{Introduction}

Teager-Kaiser Energy Operator (TKEO) (Kaiser 1990) is a local energy tracking measure of oscillatory signals that is very easy to implement efficiently. In its continuous and 1-D version, TKEO computes the energy of a real-valued signal $x(t)$ as follows:

$$
\Psi[s(t)]=\left[s^{(1)}(t)\right]^{2}-s^{(0)}(t) s^{(2)}(t)
$$

It has been shown that $\Psi$ applied to an AM-FM signal $s(t)=a(t) \cos (\omega(t))$ can approximately estimate the squared product $a^{2}(t) . \omega^{2}(t)$ assuming that $a(t)$ and $\omega(t)$ do not vary too fast or too greatly in time compared to the carrier frequency (Maragos et al. 1993a). Based on $\Psi[s(t)]$ a closed-formula for exact computation of $a(t)$ and $\omega(t)$ components called continuous Energy Separation Algorithm (ESA) is introduced (Maragos et al. 1993b). Even limited to narrow-band signals, TKEO is generally respecting the conditions of physical continuity required for the amplitude and the detected frequency (Vakman 1996). For multi-component signals analysis, TKEO requires a bandpass filtering (Havlicek et al. 2005). TKEO has been extended to the bi-dimensional (2D) signals by Yu et al. (1991). In image processing, this operator has been applied to demodulation (Maragos and Bovik 1995; Havlicek et al. 2005; Boudraa et al. 2005), noise reduction (Vleesschauwer et al. 1997), image contrast enhancement (Mitra et al. 1991; Ramponi et al. 1996; Boudraa and Diop 2008) and image thresholding (Boudraa et al. 2008). A 2D complex operator has also been developed to track the energy of oriented patterns in images (Larkin 2005). Felsberg and Jonsson (2005) have introduced energy tensors to describe the TKEO and expressed the gradient tensor by means of the Kronecker product (Felsberg and Köthe 2005). In addition, higher order generalization of TKEO called $k$-order Differential Energy Operator (DEO) $\Psi_{k}$ has been introduced by Maragos and Potamianos (1995):

$$
\Psi_{k}[s(t)]=s^{(1)}(t) s^{(k-1)}(t)-s^{(0)}(t) s^{(k)}(t)
$$

This class of operators is useful for demodulating AM-FM signals. The case $k=2$ yields the second-order DEO.

We have extended these operators to the 2D continuous signals (Boudraa et al. 2005) and demonstrated their efficiency for surface roughness detection in white light interferometry (Salzenstein et al 2005), providing an extension of the popular FSA method (Larkin 1996). Moreover, a more general expression of TKEO and higher order operators for 1D signals has been recently introduced by the authors Salzenstein et al. (2007). Recently, some authors have shown the ability of such operators to separate multi-band components (Cai et al. 2010). Finally, we have also recently introduced a generalization of some previous operators to multi-dimensional signals by means of higher order gradients and Kronecker products (Salzenstein and Boudraa 2009). In particular, we have proved that the recurrence relation introduced in Maragos and Potamianos (1995) is effective at any dimension. A limit of these operators (Maragos and Potamianos 1995; Salzenstein and Boudraa 2009) is their sensitivity in very noisy environment. 
Main contributions of this paper include:

- To overcome the sensitivity of these methods, we have introduced a new approach which is robust against noise to compute the frequency components of a n-D signal by fixing the sign of each component.

- We have introduced a new n-D scalar operator linking the directional derivatives and the gradients of a signal, including all partial derivatives at any order (Sect. 3, Proposition 1). The proofs of the proposition are reported in the Appendix section.

- We highlight, in Sect. 4, the efficiency of our approach to demodulate local AM-FM signals, provided that the scalar is proportional to a norm of the frequency vector.

- We generalize the relations provided in Maragos et al. (1993a) and Maragos and Bovik (1995) to any n-D scalar model.

- A computation of the error under such an assumption has been performed (see Appendix section).

- We have constructed a new efficient scalar operator using a directional derivative along a vector containing the local "sign" frequency components.

- The performances of the methods are investigated in presence of additive noise by extending the noise model introduced in Dimitriadis et al. (2009) to n-D signals.

We present results of demodulation of both noiseless and noisy synthetic signals, and of real data such as interferometric, Sonar and fingerprint images. To show the efficiency of our method, the results are compared to our previous operator $\Phi_{k}$ (Salzenstein and Boudraa 2009 ) and to the classical n-D operator $\Phi_{B}$ introduced by Maragos and Bovik (1995).

\section{Review of multi-dimensional TKEO and higher-order tensors of the literature}

Different operators extending TKEO to 2D signals have been developed (Maragos and Bovik 1995; Boudraa et al. 2005; Larkin 2005). These operators are useful for AM-FM signal demodulation because their outputs are proportional to the square of the product of amplitude an frequency of the input 2D signal. For 2D signal $s(\mathbf{u})$, the introduced operators denoted respectively by $\Phi_{B}$ (Maragos and Bovik 1995), $\Phi_{C}$ (Boudraa et al. 2005) and $\Phi_{D}$ (Larkin 2005) can be expressed by an energy tensor $\Psi_{t}[s(\mathbf{u})]$ (Felsberg and Granlund 2004) as follows:

$$
\Psi_{t}[s(\mathbf{u})]=[\nabla s(\mathbf{u})][\nabla s(\mathbf{u})]^{T}-s(\mathbf{u}) H s(\mathbf{u})=\left(\begin{array}{ll}
\Psi_{11} & \Psi_{12} \\
\Psi_{21} & \Psi_{22}
\end{array}\right)
$$

where

$$
\mathbf{u}=(x, y), \quad \nabla s=\left[\frac{\partial s}{\partial x} \frac{\partial s}{\partial y}\right]^{T}, \quad H=\nabla \nabla^{T}
$$

$\nabla$ and $H$ denote respectively the gradient and the Hessian of $s(\mathbf{u})$. We have the following relations:

$$
\begin{aligned}
& \Phi_{B}[s(\mathbf{u})]=\Psi_{11}+\Psi_{22}=\operatorname{Trace}\left[\Psi_{t}[s(\mathbf{u})]\right] \\
& \Phi_{C}[s(\mathbf{u})]=\Psi_{11}+\Psi_{22}+\Psi_{12}+\Psi_{21} \\
& \Phi_{D}[s(\mathbf{u})]=\Psi_{11}-\Psi_{22}+j \Psi_{12}+j \Psi_{21}
\end{aligned}
$$

We have recently introduced (Salzenstein and Boudraa 2009) a multi-dimensional approach extending both TKEO and $k$-order DEO. Let $\mathbf{u}=\left(x_{1} x_{2}, \ldots, x_{n}\right)$ be a n-D vector. For a multi-dimensional signal $s(\mathbf{u})$, the $k$ th order tensor denoted $\Psi_{k}[s(\mathbf{u})]$, is given by: 


$$
\begin{aligned}
& \Psi_{k}[s(\mathbf{u})]=\frac{d s}{d \mathbf{u}} \otimes\left(\frac{d^{k-1} s}{d \mathbf{u}^{k-1}}\right)^{T}-s \otimes\left(\frac{d^{k} s}{d \mathbf{u}^{k}}\right)^{T} \quad \text { for } k=2 p \\
& \Psi_{k}[s(\mathbf{u})]=\frac{d s}{d \mathbf{u}} \otimes\left(\frac{d^{k-1} s}{d \mathbf{u}^{k-1}}\right)-s \otimes\left(\frac{d^{k} s}{d \mathbf{u}^{k}}\right) \quad \text { for } k=2 p+1
\end{aligned}
$$

where symbol ' $\otimes$ ' denotes the Kronecker product (Moon and Stirling 2000). In particular, the trace of these tensors corresponds to $\Phi_{B}$ operator. A scalar operator $\Phi_{k}[s(\mathbf{u})]$ can be deduced by adding all elements of the matrix $\Psi_{k}[s(\mathbf{u})]$. In addition, we have shown that this class of tensors are able to track instantaneous envelope $A(\mathbf{u})$ and frequency vector $\mathbf{w}=\left(w_{1} w_{2}, \ldots, w_{n}\right)^{T}$ for any locally narrow band multi-dimensional signal such as:

$$
s\left(x_{1}, x_{2}, \ldots, x_{n}\right) \simeq A(\mathbf{u}) \cos (\mathbf{w u}+\theta)
$$

In this case we are faced with the problem of recovering the sign of the instantaneous frequency signals as pointed out in Maragos and Bovik (1995). To resolve this problem, a new "sign" component solution has been proposed by the authors Salzenstein and Boudraa (2009). However, the proposed n-D method has two limits:

- It has been applied under local sinusoidal signal assumption.

- It can fail in the presence of some frequency values.

This paper deals with three main points:

- We provide a general link between the Kronecker powers and any higher order operators.

- We generalize the relations introduced in Maragos et al. (1993a) and Maragos and Bovik (1995) related to the application of mono-dimensional and multi-dimensional TeagerKaiser (TK) operators to any local n-D AM-FM signals.

- We improve the robustness of the frequency estimation by the introduction of a new n-D scalar operator.

\section{A new generalization of TKEO by combining directional derivatives and Kronecker powers}

In this section, we introduce a new multi-dimensional operator linking higher order scalar operators using directional derivatives and Kronecker powers. Thus the N-D problem is reduced to a $1 \mathrm{D}$ one. Let $\Psi_{H_{k, p, m}}$ be a multi-dimensional tensor defined by:

$$
\Psi_{H_{k, 2 p, 2 m+1}}[s(\mathbf{u})]=\frac{d^{2 m+1} s}{d \mathbf{u}^{2 m+1}} \otimes\left(\frac{d^{2 l+1} s}{d \mathbf{u}^{2 l+1}}\right)^{T}-\frac{d^{2 p} s}{d \mathbf{u}^{2 p}} \otimes\left(\frac{d^{2 q} s}{d \mathbf{u}^{2 q}}\right)^{T}
$$

where $k=2 p+2 q=2 m+1+2 l+1$ for an even order tensor.

$$
\Psi_{H_{k, 2 p, 2 m+1}}[s(\mathbf{u})]=\frac{d^{2 m+1} s}{d \mathbf{u}^{2 m+1}} \otimes\left(\frac{d^{2 l} s}{d \mathbf{u}^{2 l}}\right)-\frac{d^{2 p} s}{d \mathbf{u}^{2 p}} \otimes\left(\frac{d^{2 q+1} s}{d \mathbf{u}^{2 q+1}}\right)
$$

where $k=2 p+2 q+1=2 m+2 l+1(m, l) \neq(p, q)$ for an odd order tensor. This corresponds to a multi-dimensional generalization of operators introduced in Salzenstein et al. (2007). According to Kronecker product properties, dimensions of the matrices are given as follows: 


$$
\begin{aligned}
\frac{d^{2 p} s}{d \mathbf{u}^{2 p}} & \Rightarrow n^{p} \text { rows } \times n^{p} \text { columns } \\
\frac{d^{2 q} s}{d \mathbf{u}^{2 q}} & \Rightarrow n^{q} \text { rows } \times n^{q} \text { columns } \\
\frac{d^{2 m+1} s}{d \mathbf{u}^{2 m+1}} & \Rightarrow n^{m} \text { rows } \times n^{m+1} \text { columns } \\
\frac{d^{2 l+1} s}{d \mathbf{u}^{2 l+1}} & \Rightarrow n^{l} \text { rows } \times n^{l+1} \text { columns }
\end{aligned}
$$

Then it follows immediately from Eqs. (4) and (5), that $\Psi_{H_{k, 2 p, 2 m+1}}$ is a $n^{m+l+1} \times n^{m+l+1}=$ $n^{p+q} \times n^{p+q}$ matrix when $k$ is an even integer and a $n^{m+l} \times n^{m+l+1}=n^{p+q} \times n^{p+q+1}$ matrix when $k$ is an odd integer.

Let us now recall the "Kronecker power" of a $n \times 1$ vector $\mathbf{v} \in \mathbb{R}^{n}$ (Vetter 1973):

$$
\bigotimes_{p} \mathbf{v}=\mathbf{v} \otimes \mathbf{v} \otimes \cdots \otimes \mathbf{v}
$$

This gives a $n^{p} \times 1$ vector. The following proposition provides the relationship between higher order tensors and higher order directional derivatives.

Proposition 1 Link between higher order even tensor Eq. (4) and all directional derivatives is given by:

$$
\left(\bigotimes_{p+q} \mathbf{v}^{\mathbf{T}}\right) \Psi_{H_{k, 2 p, 2 m+1}}[s(\mathbf{u})]\left(\bigotimes_{p+q} \mathbf{v}\right)=\frac{\partial^{2 m+1} s}{\partial \mathbf{v}^{2 m+1}}\left(\frac{\partial^{2 l+1} s}{\partial \mathbf{v}^{2 l+1}}\right)^{T}-\frac{\partial^{2 p} s}{\partial \mathbf{v}^{2 p}}\left(\frac{\partial^{2 q} s}{\partial \mathbf{v}^{2 q}}\right)^{T}
$$

In the same way, a link between higher order odd tensor Eq. (5) and all directional derivatives is given by:

$$
\left(\bigotimes_{p+q} \mathbf{v}^{\mathbf{T}}\right) \Psi_{H_{k, 2 p, 2 m+1}}[s(\mathbf{u})]\left(\bigotimes_{p+q+1} \mathbf{v}\right)=\frac{\partial^{2 m+1} s}{\partial \mathbf{v}^{2 m+1}}\left(\frac{\partial^{2 l} s}{\partial \mathbf{v}^{2 l}}\right)^{T}-\frac{\partial^{2 p} s}{\partial \mathbf{v}^{2 p}}\left(\frac{\partial^{2 q+1} s}{\partial \mathbf{v}^{2 q+1}}\right)^{T}
$$

Complete proof of the proposition is given in Appendix A and B. According to Eqs. (7-8) it is possible to construct a large class of scalar operators based on higher order tensors $\Psi_{H_{k}}$ by choosing different vectors $\mathbf{v}$.

Let us denote respectively $\Phi_{H_{k, 2 p, 2 m+1}}[s(\mathbf{u})]$ and $\Phi_{H_{k, 2 p, 2 m+1}}[s(\mathbf{u})]$ the previous operators. For example, the scalar defined in Salzenstein and Boudraa (2009) corresponds to a $n \times 1$ vector $\mathbf{v}=(1, \ldots 1)^{T}$ :

$$
\begin{array}{r}
\Phi_{2 p}[s(\mathbf{u})]=\left(\bigotimes_{p} \mathbf{v}^{\mathbf{T}}\right) \Psi_{2 p}[s(\mathbf{u})]\left(\bigotimes_{p} \mathbf{v}\right) \\
\Phi_{2 p+1}[s(\mathbf{u})]=\left(\bigotimes_{p} \mathbf{v}^{\mathbf{T}}\right) \Psi_{2 p}[s(\mathbf{u})]\left(\bigotimes_{p+1} \mathbf{v}\right)
\end{array}
$$


The particular case of the second order operator yields the directional TKEO, which extends the $1 \mathrm{D}$ classical TKEO to the directional derivatives along any unit vector:

$$
\mathbf{v}^{T} \Psi_{2}[s(\mathbf{u})]^{T} \mathbf{v}=\left(\frac{\partial s}{\partial \mathbf{v}}(\mathbf{u})\right)^{2}-s(\mathbf{u}) \frac{\partial^{2} s}{\partial \mathbf{v}^{2}}(\mathbf{u})
$$

In the next sections we use the following notation: TKEO scalar operator associated with the vector $\mathbf{v}$ and tensor $\Psi_{2}[s(\mathbf{u})]$ is denoted $\Phi_{2, \mathbf{v}}[s(\mathbf{u})]$ i.e:

$$
\mathbf{v}^{T} \Psi_{2}[s(\mathbf{u})]^{T} \mathbf{v}=\Phi_{2, \mathbf{v}}[s(\mathbf{u})]
$$

As it will be seen in the next section, an interesting application of this method is the AM-FM signal demodulation.

\section{Demodulation of $n-D$ signal}

In this section, we present an envelope and frequency demodulation method of any local AM-FM signal (Sect. 4.1). In particular, the general condition related to the choice of a directional derivative, which leads to a new stable and robust method (Sect. 4.2) is provided.

\subsection{Instantaneous envelope and frequency demodulation}

Let $s$ be an $n$-dimensional an AM-FM signal defined by:

$$
s(\mathbf{u})=A(\mathbf{u}) \cos (\phi(\mathbf{u}))
$$

This signal must have smooth local coherency in order to ensure that a particular pair of AM-FM components can be selected (Bovik et al. 1992). When the envelop $A$ (u) does not vary too fast compared to the carrier, the gradient of the phase is such that $\nabla \phi=\mathbf{w}(\mathbf{u}) \simeq \mathbf{w}$, the local frequency vector is given by $\mathbf{w}=\left(w_{1} w_{2}, \ldots, w_{n}\right)^{T}$. We choose a normalized vector $\mathbf{v}=\left(v_{1} v_{2}, \ldots, v_{n}\right)^{T}$ so that (see next section):

$$
\frac{\partial \phi}{\partial \mathbf{v}}=\frac{d \phi}{d \mathbf{u}} \mathbf{v}=\mathbf{w}^{T} \mathbf{v} \geq\|w\|
$$

This assumption leads to the following approximation (see also Appendix C):

$$
\Phi_{2, \mathbf{v}}[s(\mathbf{u})] \simeq A(\mathbf{u})^{2}\left(\frac{\partial \phi}{\partial \mathbf{v}}(\mathbf{u})\right)^{2}=A(\mathbf{u})^{2}\left(\mathbf{w}(\mathbf{u})^{T} \mathbf{v}\right)^{2}
$$

Thus, the extracted envelope is given by

$$
A(\mathbf{u})^{2} \simeq \frac{\left|\Phi_{2, \mathbf{v}}^{2}[s(\mathbf{u})]\right|}{\left|\Phi_{2, \mathbf{v}}^{2}\left[\frac{\partial s(\mathbf{u})}{\partial \mathbf{v}}\right]\right|}=\frac{\left|\Phi_{2, \mathbf{v}}^{2}[s(\mathbf{u})]\right|}{\left|\Phi_{4, \mathbf{v}}^{2}[s(\mathbf{u})]\right|}
$$

Hence, Eq. (7) is helpful to compute the scalar $\Phi_{4, \mathbf{v}}^{2}[s(\mathbf{u})]$. Such results can be easily extended to any higher order operators and computed by using Eqs. (7-8). The instantaneous frequency vector components are estimated using the algorithm developed in Salzenstein and Boudraa (2009).

Let us consider the derivative signal $s_{i_{1} x_{1}, i_{2} x_{2}, \ldots, i_{n} x_{n}}(\mathbf{u})$ obtained by the partial derivatives along $x_{i}$.

$$
s_{i_{1} x_{1}, i_{2} x_{2}, \ldots, i_{n} x_{n}}(\mathbf{u})=i_{1} \frac{\partial s}{\partial x_{1}}+i_{2} \frac{\partial s}{\partial x_{2}}+\cdots+i_{n} \frac{\partial s}{\partial x_{n}}
$$


where $i_{j}$ are integers. One can write using (15):

$$
\left(i_{1} w_{1}+i_{2} w_{2}+\cdots+i_{n} w_{n}\right)^{2} \simeq \frac{\Phi_{2, \mathbf{v}}\left[s_{i_{1} x_{1}, i_{2} x_{2}, \ldots, i_{n} x_{n}}(\mathbf{u})\right]}{\Phi_{2, \mathbf{v}}[s(\mathbf{u})]}
$$

As in Salzenstein and Boudraa (2009) it is then possible to extract a unique frequency vector. We call $\mathbf{w}^{(\mathbf{j})}=\left(w_{1}^{(j)}, \ldots, w_{n}^{(j)}\right)$ the set of components obtained by fixing the sign $\epsilon_{j}= \pm 1$ of a chosen component $w_{j}=\epsilon_{j}\left|w_{j}\right|(\neq 0)$ :

1. Compute the quantities:

$$
\begin{aligned}
\left|w_{j}\right| & =\sqrt{\frac{\Phi_{2, \mathbf{v}}\left[s_{x_{j}}\right]}{\Phi_{2, \mathbf{v}}[s]}} \\
w_{j} w_{k} & =\frac{\Phi_{2, \mathbf{v}}\left[s_{x_{j}}+s_{x_{k}}\right]-\Phi_{2, \mathbf{v}}\left[s_{x_{j}}-s_{x_{k}}\right]}{4 \Phi_{2, \mathbf{v}}[s]}
\end{aligned}
$$

2. Compute the $j$ th component:

$$
w_{j}^{(j)}=\epsilon_{j} \operatorname{sign}\left(w_{j} w_{j}\right)\left|w_{j}\right|=\epsilon_{j}\left|w_{j}\right|
$$

3. Compute of the $k$ th component, $k \in\{1, \ldots, n\}$ :

$$
w_{k}^{(j)}=\epsilon_{j} \operatorname{sign}\left(w_{k} w_{j}\right)\left|w_{k}\right|
$$

4.2 Choice of the directional derivatives

The recent model introduced in Salzenstein and Boudraa (2009) corresponds to the directional derivatives along the vector $\mathbf{v}=(1,1, \ldots, 1)^{T}$. However, this algorithm can fail when the sum of the frequency components is null:

$$
\mathbf{w}^{T} \mathbf{v}=w_{1}+w_{2}+\cdots+w_{n}=0
$$

To ensure the stability of the method, the following condition is necessary :

$$
\Phi_{2, \mathbf{v}}[A(\mathbf{u}) \cos (\mathbf{w u}+\theta)]=0 \Leftrightarrow \mathbf{w} \simeq \mathbf{0}
$$

We have added a second condition in order to minimize the error bound:

$$
\frac{\partial \phi}{\partial \mathbf{v}}=\frac{d \phi}{d \mathbf{u}} \mathbf{v}=\mathbf{w}^{T} \mathbf{v} \geq\|w\|
$$

In other words, any local AM-FM signal applied to a generalized scalar operator $\Phi_{2, \mathbf{v}}[s(\mathbf{u})]$ should be proportional or greater to a norm of the frequency vector. The image demodulation technique developed in Maragos and Bovik (1995) corresponds to the usual Euclidean quadratic norm. In this paper, we propose a new scalar multi-dimensional algorithm which compares favorably to the previous ones, using local directional derivatives according to the normalized vector

$$
\mathbf{v}(\mathbf{u}) \propto \operatorname{sign}(\mathbf{w})=\left(\operatorname{sign}\left(w_{1}\right) \operatorname{sign}\left(w_{2}\right), \ldots, \operatorname{sign}\left(w_{n}\right)\right)^{T}
$$

at any point $\mathbf{u}$. Applying Eq. (14) leads immediately to:

$$
\Phi_{2, \operatorname{sign}(\mathbf{w})}[s(\mathbf{u})] \simeq \frac{A^{2}}{n}\left(\sum\left|w_{i}\right|\right)^{2}
$$


The main problem lies in the recovering of the sign of the local frequency components. To overcome this problem, calculus of Eq. (22) is done in another way. Actually, the general term of the matrix $\Psi_{2}[s(\mathbf{u})]$ computed by the relation (4) equals:

$$
[\Psi]_{i j} \simeq A^{2} w_{i}^{k_{i}} w_{j}^{k_{j}} \quad k_{i}+k_{j}=2
$$

Thus adding all absolute values of the elements of the matrix provides the final expression:

$$
\sum_{i, j}\left|[\Psi]_{i j}\right|=A^{2}\left(\left|w_{1}+\cdots+\right| w_{n} \mid\right)^{2}
$$

which is proportional to the general scalar operator (22) using a directional derivative along the vector $\mathbf{v}(\mathbf{u})=\left(\operatorname{sign}\left(w_{1}\right) \operatorname{sign}\left(w_{2}\right), \ldots, \operatorname{sign}\left(w_{n}\right)\right)^{T}$ at any point $\mathbf{u}$. Finally we apply the following general algorithm to demodulate local AM-FM signal:

1. Compute the matrix $\Psi_{H_{k, 2 p, 2 m+1}}[s(\mathbf{u})]$ using the relation (4) for an even order $k=2$ (resp. $k=4$ ) and the associated parameters $p, q, m, l$ (resp. $p 1, q 1, m 1, l 1$ ) provided that $k=2 p+2 q=2 m+1+2 l+1$ (resp. $2 k=2 p 1+2 q 1=2 m 1+1+2 l 1+1)$

2. Compute the sum of all absolute values of the elements of the matrix;

3. Compute the instantaneous envelope using Eq. (15);

4. Compute the instantaneous frequency components using the "sign" algorithm outlined at the end of the Sect. 4.1;

This algorithm corresponding to the operator $\Psi_{2, \operatorname{sign}(\mathbf{w})}$ is called Algo 1.

\subsection{An alternative algorithm using eigenvectors}

We show in this subsection that there is an alternative way to compute the sign of the frequency components. Actually, the general term of the tensor matrix $\Psi_{2}$ is approximatively equal to $A^{2} w_{i} w_{j}$ where $w_{i}$ and $w_{j}$ are respectively the $i$ th and $j$ th component of the frequency vector (i.e, the gradient of the phase). We immediately observe that the normalized frequency vector:

$$
\mathbf{v}=\frac{\mathbf{w}}{\|\mathbf{w}\|}
$$

is an eigenvector associated with the eigenvalue $\lambda=A^{2}\|\mathbf{w}\|^{2}$. Although this eigenvector does not provide the absolute frequency vector, it gives the relative sign of the frequency components, i.e., the local orientation. On the other hand, $\Psi_{2}$ is a symmetric matrix, which defines a positive semi-definite quadratic form for any narrow band signal i.e.,:

$$
\forall \mathbf{v} \neq \mathbf{0} \quad \mathbf{v}^{T} \Psi_{2}[s] \mathbf{v} \geq 0
$$

According to the properties of such forms, it follows that its eigenvalues are positive or null (Moon and Stirling 2000). Moreover, the trace of the tensor equalling $A^{2}\|\mathbf{w}\|^{2}$ it follows immediately that $\lambda=A^{2}\|\mathbf{w}\|^{2}$ corresponds to the maximum eigenvalue of $\Psi_{2}$ (the two other eigenvalues being null, showing that the tensor is a singular matrix). Thus instead of the step 4 in Algo 1, we propose the following algorithm in order to compute the frequency vector:

1. Compute the quantities:

$$
\left|w_{j}\right|=\sqrt{\frac{\Phi_{2, \mathbf{v}}\left[s_{x_{j}}\right]}{\Phi_{2, \mathbf{v}}[s]}}
$$


2. Compute the eigenvector $\mathbf{v}$ of the tensor $\Psi_{2}$ associated with its maximum eigenvalue.

3. Compute of the $j$ th component, $j \in\{1, \ldots, n\}$ :

$$
w_{j}=\operatorname{sign}\left(v_{j}\right)\left|w_{j}\right|
$$

This algorithm is referred to as Algo 2. This step only focuses on the frequency estimation, for the envelope detection being the procedure is identical to Algo 1. Let us remark that the scalar operator $\Phi_{2, \mathbf{v}}$ associated with the eigenvector $\mathbf{v}$ gives the trace of $\Psi_{2}$ i.e., an operator which is comparable to $\Phi_{B}$.

\section{Results and discussions}

To show the efficiency of our method, the results of synthetic and real data (Interferometry, Sonar and fingerprints) are compared to our previous operator $\Phi_{k}$ (Salzenstein and Boudraa 2009 ) and to the classical n-D operator $\Phi_{B}$ introduced by Maragos and Bovik (1995). This operator corresponds to the trace of the $\Psi_{2}$ tensor. The output of $\Phi_{B}$ to a $n$-dimensional AM-FM signal with slowly varying amplitude and frequencies is given by Maragos and Bovik (1995):

$$
\Phi_{B}[s(\mathbf{u})]=A^{2}\left(w_{1}^{2}+w_{2}^{2}+\cdots+w_{n}^{2}\right)
$$

For narrow band AM-FM signal, the envelope and the modulus of the frequency components can be approximated by the following relations:

$$
A^{2} \approx \frac{\Phi_{B}[s(\mathbf{u})]}{\sum_{k=1}^{n} \Phi_{B}\left[s_{x_{k}}\right]} \quad\left(w_{k}\right)^{2} \approx \frac{\Phi_{B}\left[s_{x_{k}}\right]}{\Phi_{B}[s(\mathbf{u})]}
$$

Thus, $\Phi_{B}$ based demodulation requires derivatives until the third order. While operator $\Phi_{k}$ (Salzenstein and Boudraa 2009) requires partial derivatives until the fourth order, the new multi-dimensional scalar operator Eq. (4) is calculated for respective orders $k=2$ and $k=4$ (Step 1 of Sect. 4.2). These functions also require derivatives until the third order. Note that, although in Maragos and Bovik (1995) the problem of relative sign of the frequency components has not been studied, a possible solution to this problem is to replace $\Psi_{2, \mathbf{v}}$ by $\Phi_{B}$ in Eqs. (25) and (19). We compare Algo 1 and Algo 2 to $\Phi_{B}$ and $\Phi_{k}$ operators on 2D and 3D data in terms of instantaneous envelope and frequency components extraction. Comparison with other multi-dimensional methods have been reported in Salzenstein and Boudraa (2009) where the 3D approaches i.e, the algorithms $\Phi_{B}$ and $\Phi_{k}$ give best results. The relative error rates related to the original data (if available) are calculated at each point $\mathbf{u}=\left(\begin{array}{lll}x & y & z\end{array}\right)$ as follows:

$$
\varepsilon_{e n v}=\frac{|A(\mathbf{u})-\hat{A}(\mathbf{u})|}{A(\mathbf{u})} \quad \varepsilon_{f r e q}=\frac{\|\mathbf{w}(\mathbf{u})-\hat{\mathbf{w}}(\mathbf{u})\|}{\|\mathbf{w}(\mathbf{u})\|}
$$

Different slices of a synthetic fringe pattern $(128 \times 128 \times 128)$ where the envelope and the local carrier waves are varying according to the directions and magnitudes of the frequency vectors are presented in Figs. 1 and 2. Error rates of envelope and frequency estimates given by all methods for the two synthetic data in noise free and noisy environments are summarized in Tables 1, 2, 3, 4, 5 and 6. Tables 1 and 2 report the results for noise free data while Tables 3-4 and Tables 5 and 6 summarize the results with respective noise levels of 10 and $20 \%$. Globally, the new operator $\Psi_{2 \text {,sign(w) }}$ (Algo 1) gives a better error rate than the other multi-dimensional energy separation algorithms. The improvement in frequency detection in 


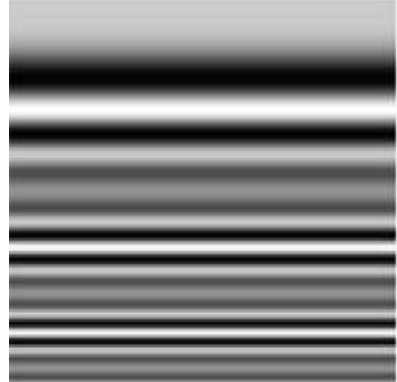

(a)

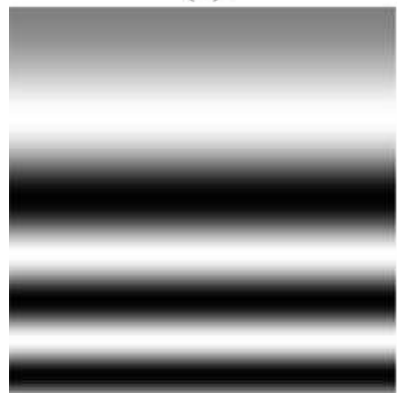

(d)

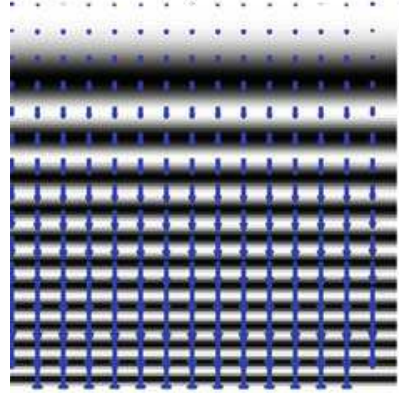

(g)
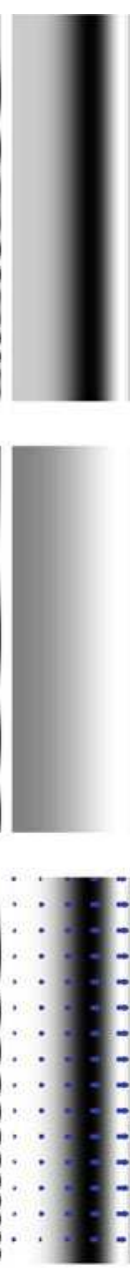

.
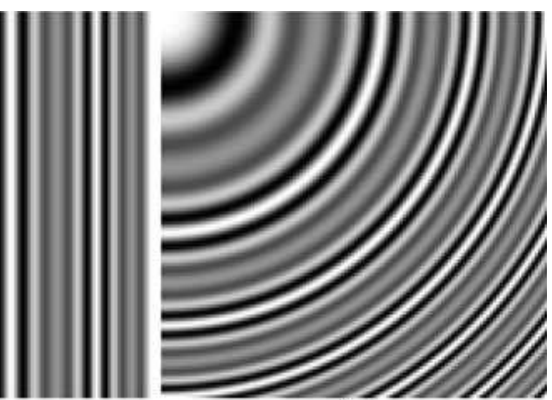

(b)

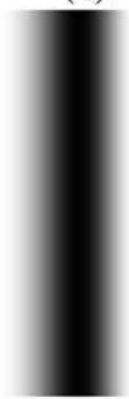

(e)
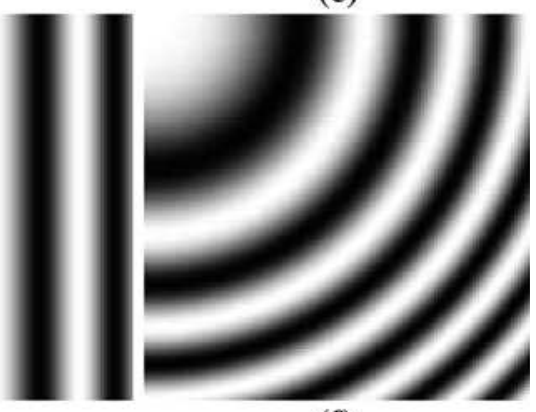

(h)

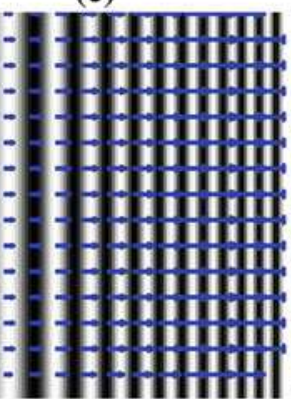

(f)

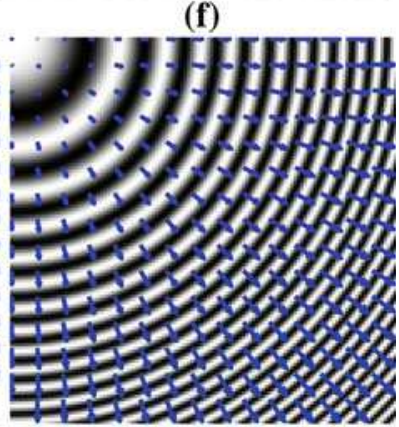

(i)

Fig. 1 Contrasted pattern (I) with a contrasted envelope. a xy slice; b yz slice; $\mathbf{c} x z$ slice; $\mathbf{d}$ xy envelope; e yz envelope; $\mathbf{f}$ xz envelope; $\mathbf{g}$ xy frequency and carrier; $\mathbf{h}$ yz frequency and carrier; $\mathbf{i}$ xz frequency and carrier

noisy environment is particularly interesting, as illustrated by the results reported by Table 6. In 3D noisy context, the frequency detection using the eigenvector (Algo 2) gives the worst error rates. Other experimental results performed on 2D real data in both noisy and free noise context confirm the high performance of the method $\Psi_{2, \operatorname{sign}(\mathbf{w})}$ (Algo 1) regarding the local frequency detection. Actually, Figs. 3 and 4 illustrate the frequency component detection in a context of a noisy/free noise images. Sonar images and fingerprint patterns (extracted from the database ${ }^{1}$ ) are represented in Figs. 3 and 4. Sonar images show ripples and ridges of sand where their orientation and frequency oscillations is very useful information for sea-bed characterization. We have focused on the quantitative performances of the operators related to the frequency components. The frequency vectors extracted by $\Phi_{B}$ is more visually degraded specifically in the noisy context (see Figs. 3c,f, 4e,f). Tables 7 and 8

1 http://bias.csr.unibo.it/fvc2000/download.asp. 


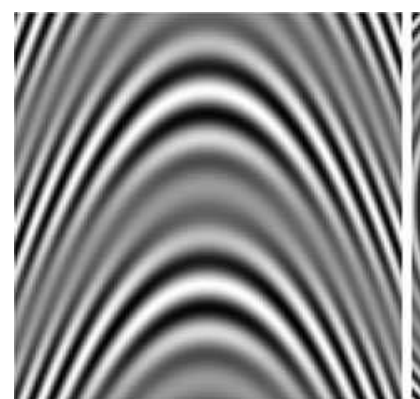

(a)

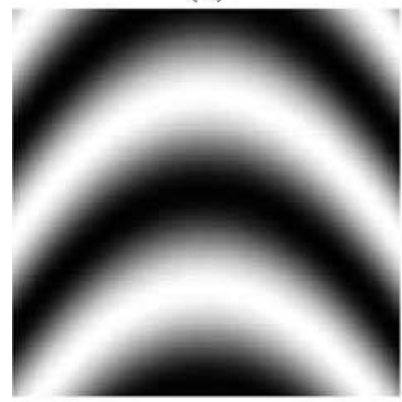

(d)

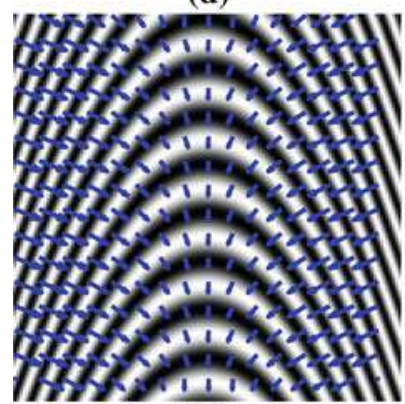

(g)

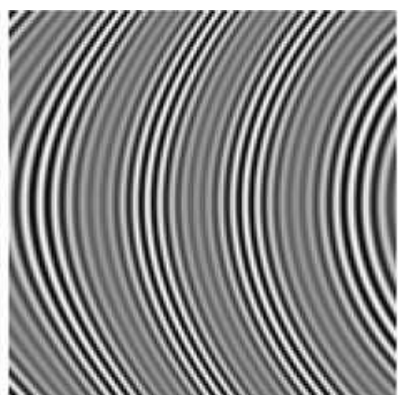

(b)

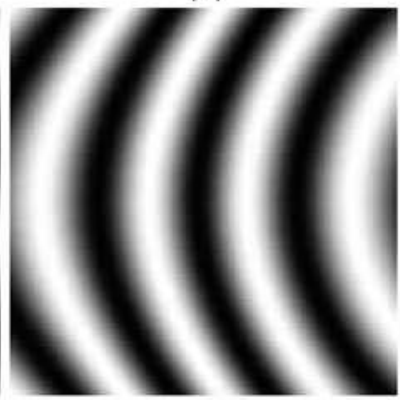

(e)

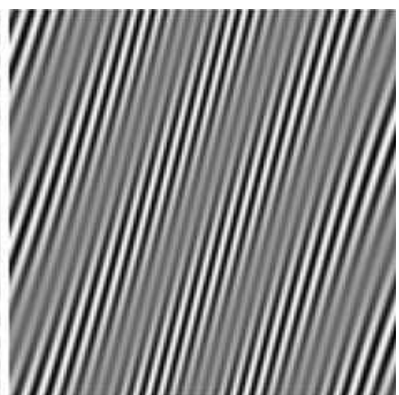

(c)

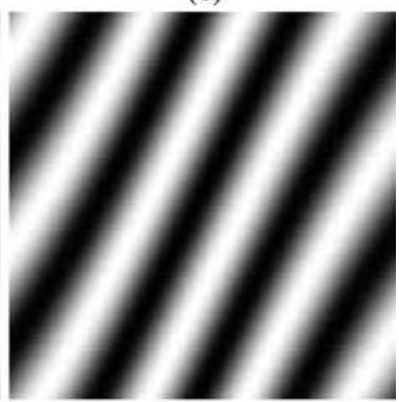

(f)

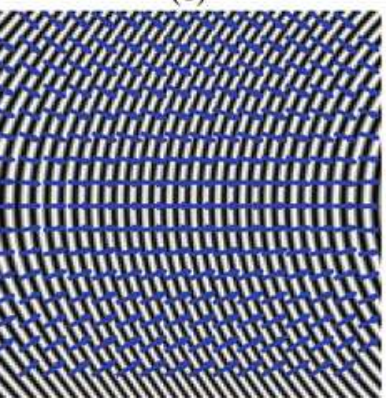

(h)

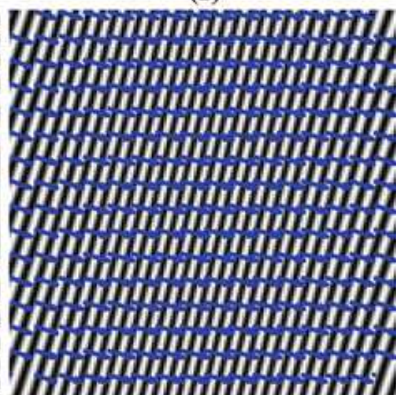

(i)

Fig. 2 Contrasted pattern (II) with a contrasted envelope. a xy slice; $\mathbf{b} y z$ slice; $\mathbf{c} x z$ slice; $\mathbf{d}$ xy envelope; e yz envelope; $\mathbf{f}$ xz envelope; $\mathbf{g}$ xy frequency and carrier; $\mathbf{h}$ yz frequency and carrier; $\mathbf{i} x \mathrm{z}$ frequency and carrier

Table 1 Global envelope error rates for noise-free patterns

\begin{tabular}{lll}
\hline Operators & $\begin{array}{l}\text { Contrast pattern I } \\
(\text { Fig. 1) }(\%)\end{array}$ & $\begin{array}{l}\text { Contrast pattern II } \\
\text { (Fig. 2) }(\%)\end{array}$ \\
\hline $3 \mathrm{D} \Phi_{2, \operatorname{sign}(\mathbf{w})}$ & 1.73 & 0.16 \\
$\mathrm{D} \Phi_{k}$ & 1.73 & 0.49 \\
$3 \mathrm{D} \Phi_{B}$ & 2.22 & 0.32 \\
\hline
\end{tabular}

also summarize the quantitative results corresponding to the relative error rates between the frequency detection in the noise free context and the one in the noisy context, for different

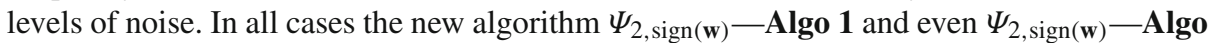
2 show their effective robustness, providing good error rates. As we have chosen a unique reference for the comparison with the noise free context $\left(\Psi_{2, \operatorname{sign}(\mathbf{w})}(\mathbf{A l g o} \mathbf{1})\right.$ or $\left.\Phi_{B}\right)$ or a reference with regard to each algorithm, the levels of error rates remain unchanged. 
Table 2 Global frequency error rates for noise-free patterns

Table 3 Global envelope error rates for $10 \%$ noisy patterns

Table 4 Global frequency error rates for $10 \%$ noisy patterns

Table 5 Global envelope error rates for $20 \%$ noisy patterns

Table 6 Global frequency error rates for $20 \%$ noisy patterns

\begin{tabular}{lll}
\hline Operators & $\begin{array}{l}\text { Contrast pattern } \\
\text { I }(\%)\end{array}$ & $\begin{array}{l}\text { Contrast pattern } \\
\text { II }(\%)\end{array}$ \\
\hline 3D $\Phi_{2, \operatorname{sign}(\mathbf{w})-\text { Algo } 1}$ & 2.61 & 3.48 \\
$3 \mathrm{D} \Phi_{2, \operatorname{sign}(\mathbf{w})-\text { Algo } 2}$ & 2.61 & 6.54 \\
$3 \mathrm{D} \Phi_{k}$ & 2.63 & 3.48 \\
$3 \mathrm{D} \Phi_{B}$ & 2.63 & 3.48 \\
\hline
\end{tabular}

\begin{tabular}{lll}
\hline Operators & $\begin{array}{l}\text { Contrast pattern I } \\
(\text { Fig. 1) }(\%)\end{array}$ & $\begin{array}{l}\text { Contrast pattern II } \\
\text { (Fig. 2) }(\%)\end{array}$ \\
\hline $3 \mathrm{D} \Phi_{2, \operatorname{sign}(\mathbf{w})}$ & 8.29 & 6.39 \\
$3 \mathrm{D} \Phi_{k}$ & 7.61 & 6.48 \\
$3 \mathrm{D} \Phi_{B}$ & 9.27 & 6.62 \\
\hline
\end{tabular}

\begin{tabular}{lll}
\hline Operators & $\begin{array}{l}\text { Contrast pattern } \\
\text { I }(\%)\end{array}$ & $\begin{array}{l}\text { Contrast pattern } \\
\text { II }(\%)\end{array}$ \\
\hline 3D $\Phi_{2, \operatorname{sign}(\mathbf{w})-\text { Algo } 1}$ & 3.25 & 3.57 \\
$3 \mathrm{D} \Phi_{2, \operatorname{sign}(\mathbf{w})}-$ Algo 2 & 13.24 & 8.24 \\
3D $\Phi_{k}$ & 4.42 & 3.93 \\
$3 \mathrm{D} \Phi_{B}$ & 4.38 & 3.72 \\
\hline
\end{tabular}

\begin{tabular}{lll}
\hline Operators & $\begin{array}{l}\text { Contrast pattern I } \\
(\text { Fig. 1) }(\%)\end{array}$ & $\begin{array}{l}\text { Contrast pattern II } \\
(\text { Fig. 2) }(\%)\end{array}$ \\
\hline $3 \mathrm{D} \Phi_{2, \operatorname{sign}(\mathbf{w})}$ & 14.13 & 12.15 \\
$3 \mathrm{D} \Phi_{k}$ & 13.96 & 12.51 \\
$3 \mathrm{D} \Phi_{B}$ & 16.09 & 12.84 \\
\hline
\end{tabular}

\begin{tabular}{|c|c|c|}
\hline Operators & $\begin{array}{l}\text { Contrast pattern } \\
\text { I }(\%)\end{array}$ & $\begin{array}{l}\text { Contrast pattern } \\
\text { II }(\%)\end{array}$ \\
\hline $3 \mathrm{D} \Phi_{2, \operatorname{sign}(\mathbf{w})}-\mathrm{Algo} 1$ & 4.98 & 3.95 \\
\hline $3 \mathrm{D} \Phi_{2, \operatorname{sign}(\mathbf{w})}-\mathrm{Algo} 2$ & 13.8 & 8.21 \\
\hline $3 \mathrm{D} \Phi_{k}$ & 12.81 & 6.72 \\
\hline $3 \mathrm{D} \Phi_{B}$ & 10.01 & 5.12 \\
\hline
\end{tabular}

\section{Comparison of $\Psi_{2}, \operatorname{sign}(w)$ and $\Phi_{B}$ in additive noise: theoretical and empirical approaches}

\subsection{Noise model}

To validate and analyze more deeply the previous results, we propose a general scheme to compare theoretically the multi-dimensional operator $\Phi_{2 \text {,sign(w) }}$ developed in this paper with the well known multi-dimensional $\Phi_{B}$ operator (Maragos and Bovik 1995) in presence of noisy additive components and different frequencies. In order to overcome the 


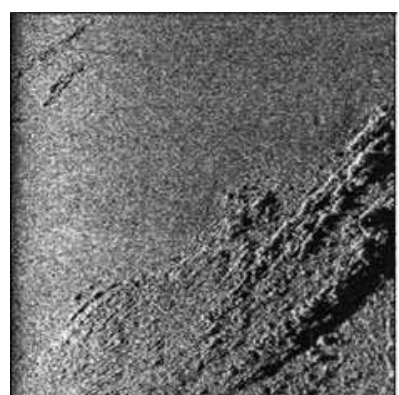

(a)

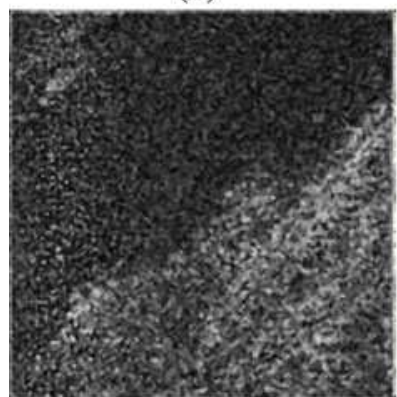

(c)

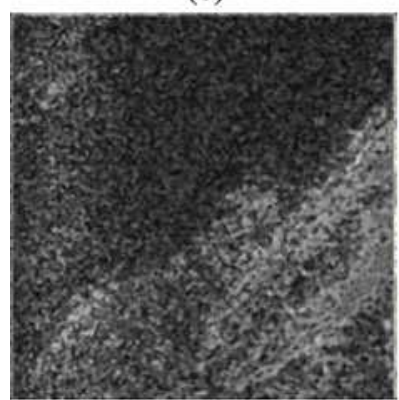

(f)

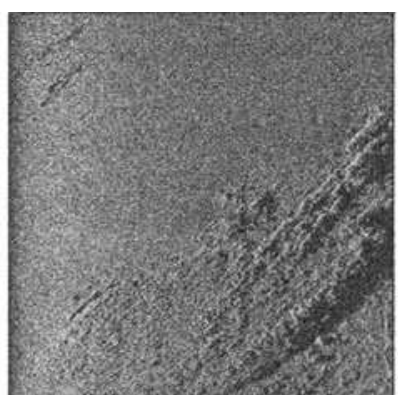

(b)

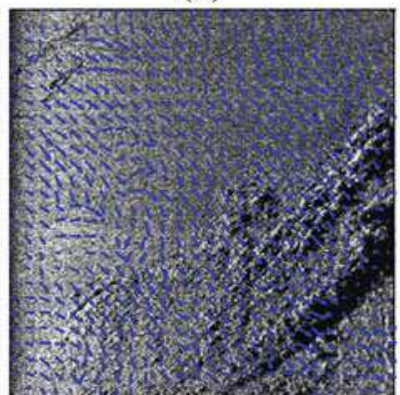

(d)

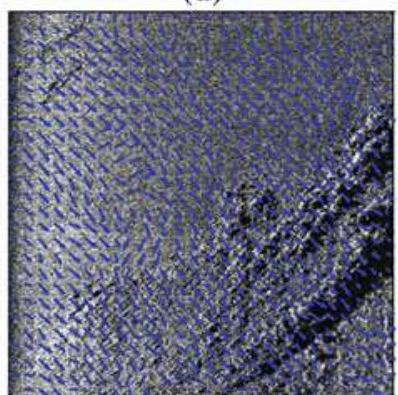

(g)

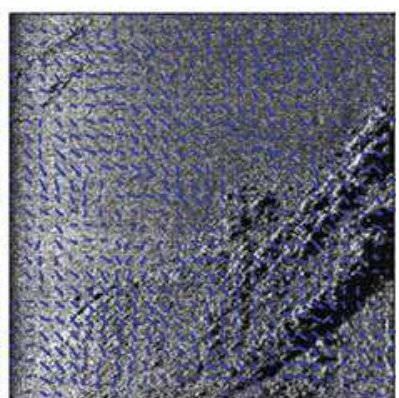

(e)

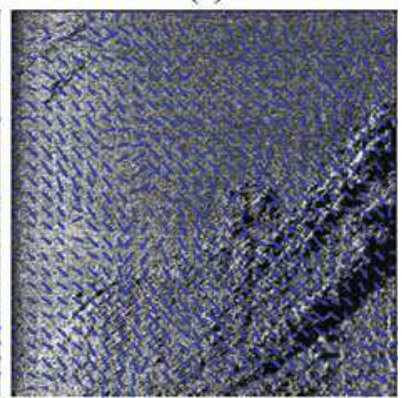

(h)

Fig. 3 Sonar image. a Free noise original slice; b $20 \%$ noisy original image; c estimated envelope by $\Phi_{B}$; d free noise estimated frequency by $\Phi_{B}$; e $20 \%$ noisy estimated frequency by $\Phi_{B}$; f estimated envelope by $\Phi_{H_{k}} ; \mathbf{g}$ free noise estimated frequency by $\Phi_{H_{k}} ; \mathbf{h} 20 \%$ noisy estimated frequency by $\Phi_{H_{k}}$

complexity and non linearity of the problem, we use the same strategy as the approach proposed in Dimitriadis et al. (2009), where the authors have investigated the signal to noise ratio related to the mono-dimensional TK and a short term energy signal operator. For that purpose, they have based their study on an approximation concerning a wide range of noise models obtained by adding $K$ sinusoids containing random phase offsets. We suggest adapting this model to the multi-dimensional context. Let us define a narrow band noisy signal $x(\mathbf{u})=s(\mathbf{u})+n(\mathbf{u})$, where $s(\mathbf{u})$ represents a clean multi-dimensional AM-FM narrow band signal and $n(\mathbf{u})$ an uncorrelated noisy component. As in Dimitriadis et al. (2009), let us define the multi-dimensional noise signal by the means of $K$ sinusoids: 


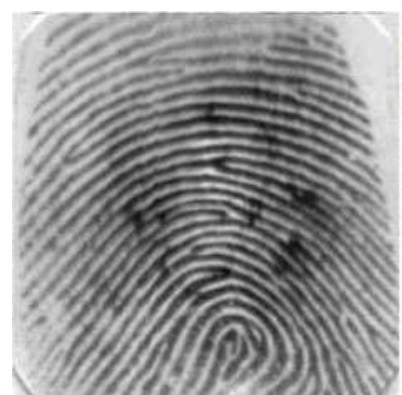

(a)

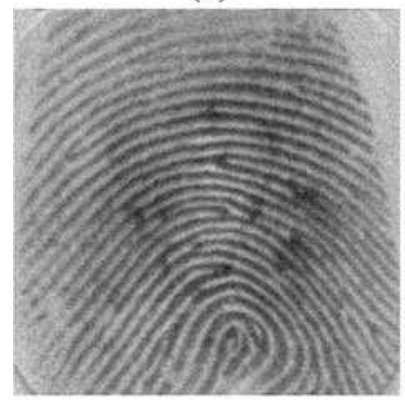

(d)

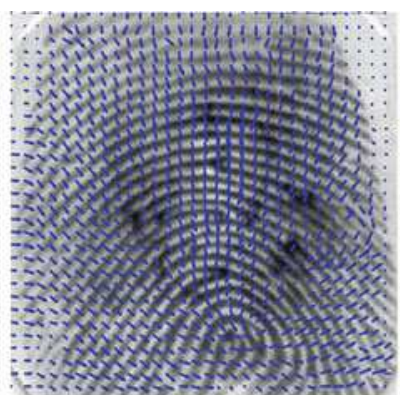

(b)

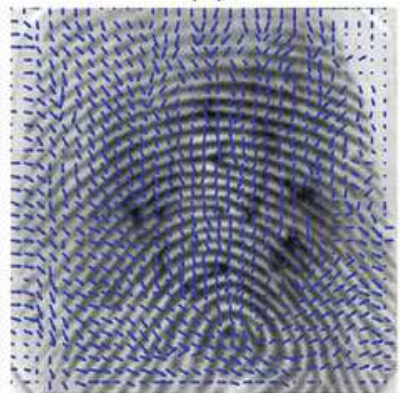

(e)

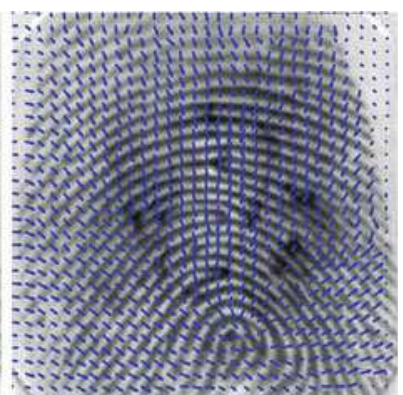

(c)

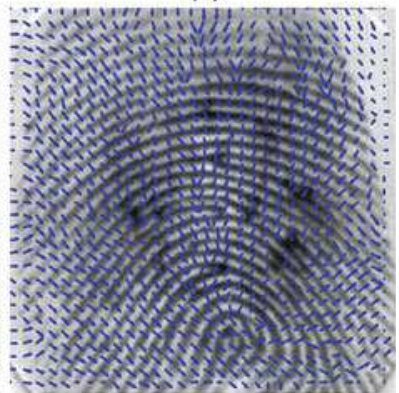

(f)

Fig. 4 Fingerprints: a original image; b estimated frequency by $\Phi_{B} ; \mathbf{c}$ estimated frequency by $\Phi_{H_{k}} ; \mathbf{d}$ noisy image; e estimated frequency by $\Phi_{B} ; \mathbf{f}$ estimated frequency by $\Phi_{H_{k}}$

$$
n(\mathbf{u})=\sum_{i=1}^{K} B_{i} \cos \left(\eta_{i}^{T} \mathbf{u}+\boldsymbol{\theta}_{i}\right)
$$

where the random offsets $\boldsymbol{\theta}_{i}$ are uniformly distributed over $[\pi,+\pi]$ and the frequency components are distinct from each other i.e., $\eta_{i} \neq \eta_{j} \forall i \neq j$. We will define the long-term time average $<f(u)>$ in the multi-dimensional context by the $\mathrm{n}-\mathrm{D}$ integration of the signal over a pavement $D_{\mathrm{T}}=\left[-T_{1} / 2, T_{1} / 2\right] \times\left[-T_{2} / 2, T_{2} / 2\right] \times \cdots \times\left[T_{n} / 2, T_{n} / 2\right]$ where the surface of $D_{\mathbf{T}}$ exceeds the largest signal period:

$$
<f(u)>=\frac{1}{T_{1} T_{2}, \ldots, T_{n}} \oint_{D_{\mathbf{T}}} f(\mathbf{u}) d \mathbf{u}
$$

Moreover, let us define the ratio $R_{B}$ and $R_{k}$ :

$$
\begin{aligned}
R_{B} & =\frac{\left\langle\Phi_{B}[n(\mathbf{u})]\right\rangle}{\left\langle\Phi_{B}[s(\mathbf{u})]\right\rangle} \\
R_{k} & =\frac{\left\langle\Phi_{2, \operatorname{sign}(\mathbf{w})}[n(\mathbf{u})]\right\rangle}{\left\langle\Phi_{2, \operatorname{sign}(\mathbf{w})}[s(\mathbf{u})]\right\rangle}
\end{aligned}
$$

They represent the contribution of the noise over the clean estimate called energy noisy ratio (ENR). In order to study the behavior of $\Phi_{B}$ and $\Phi_{2, \operatorname{sign}(\mathbf{w})}$ in a noisy context, we investigate the previous quantities and apply them to different narrow band signals containing different frequency components. 
Table 7 Global frequency error rates for $10 \%$ noisy patterns

\begin{tabular}{lll}
\hline Operators & Sonar image (\%) & Fingerprint (\%) \\
\hline 2D $\Phi_{2, \operatorname{sign}(\mathbf{w})-A l g o} 1$ & 3.67 & 2.53 \\
2D $\Phi_{2, \operatorname{sign}(\mathbf{w})}$-Algo 2 & 2.97 & 1.80 \\
$2 \mathrm{D} \Phi_{k}$ & 9.73 & 8.07 \\
$2 \mathrm{D} \Phi_{B}$ & 12.58 & 7.94 \\
\hline
\end{tabular}

\begin{tabular}{lll}
\hline Operators & Sonar image (\%) & Fingerprint (\%) \\
\hline 2D $\Phi_{2, \operatorname{sign}(\mathbf{w})}$-Algo 1 & 5.98 & 4.97 \\
$2 \mathrm{D} \Phi_{2, \operatorname{sign}(\mathbf{w})}-$ Algo 2 & 5.52 & 4.54 \\
$2 \mathrm{D} \Phi_{k}$ & 14.86 & 13.82 \\
$2 \mathrm{D} \Phi_{B}$ & 14.97 & 18.64 \\
\hline
\end{tabular}

Table 8 Global frequency error rates for $20 \%$ noisy patterns

\subsection{Expression of the ENR}

Let us now express the ENR as defined in the previous section. We base our assumption on nearly local sinusoidal signal i.e $s(\mathbf{u}) \simeq A \cos \left(\mathbf{w}^{T} \mathbf{u}\right)$. According to our results $\Phi_{2, \mathbf{v}}[s(\mathbf{u})]=$ $A^{2}\left(\mathbf{w}^{T} \mathbf{v}\right)^{2}$. Let us compute now $\Phi_{2, \mathbf{v}}[n(\mathbf{u})]$ : we have defined $n(\mathbf{u})=\sum_{i} n_{i}(\mathbf{u})$, where $n_{i}(\mathbf{u})=B_{i} \cos \left(\boldsymbol{\eta}_{i}^{T} \mathbf{u}+\boldsymbol{\theta}_{i}\right)$. Applying the operator to a sum of function leads to:

$$
\begin{aligned}
\Phi_{2, \mathbf{v}}[n(\mathbf{u})]= & \sum_{i} \Phi_{2, \mathbf{v}}\left[n_{i}(\mathbf{u})\right]+\sum_{i} \sum_{j \neq i}\left(\frac{\partial n_{i}}{\partial \mathbf{v}} \frac{\partial n_{j}}{\partial \mathbf{v}}-n_{i} \frac{\partial^{2} n_{j}}{\partial \mathbf{v}^{2}}\right) \\
= & \sum_{i} B_{i}^{2}\left(\boldsymbol{\eta}_{i}^{T} \mathbf{v}\right)^{2}+\sum_{i} \sum_{j \neq i} B_{i} B_{j}\left(\eta_{i}^{T} \mathbf{v}\right)\left(\eta_{j}^{T} \mathbf{v}\right) \sin \left(\eta_{i}^{T} \mathbf{u}+\boldsymbol{\theta}_{i}\right) \sin \left(\boldsymbol{\eta}_{j}^{T} \mathbf{u}+\boldsymbol{\theta}_{j}\right) \\
& -\sum_{i} \sum_{j \neq i} B_{i} B_{j}\left(\eta_{j}^{T} \mathbf{v}\right)^{2} \cos \left(\eta_{i}^{T} \mathbf{u}+\boldsymbol{\theta}_{i}\right) \cos \left(\eta_{j}^{T} \mathbf{u}+\boldsymbol{\theta}_{j}\right) \\
= & \sum_{i} B_{i}^{2}\left(\eta_{i}^{T} \mathbf{v}\right)^{2}+\frac{1}{2} \sum_{i} \sum_{j \neq i} B_{i} B_{j}\left(\eta_{j}^{T} \mathbf{v}\right)\left(\eta_{i}^{T} \mathbf{v}-\eta_{j}^{T} \mathbf{v}\right) \\
& \times \cos \left(\eta_{i}^{T} \mathbf{u}+\boldsymbol{\theta}_{i}-\boldsymbol{\eta}_{j}^{T} \mathbf{u}-\boldsymbol{\theta}_{j}\right) \\
& -\frac{1}{2} \sum_{i} \sum_{j \neq i} B_{i} B_{j}\left(\eta_{j}^{T} \mathbf{v}\right)\left(\eta_{i}^{T} \mathbf{v}+\eta_{j}^{T} \mathbf{v}\right) \cos \left(\eta_{i}^{T} \mathbf{u}+\boldsymbol{\theta}_{i}+\boldsymbol{\eta}_{j}^{T} \mathbf{u}+\boldsymbol{\theta}_{j}\right)
\end{aligned}
$$

The assumption that the long-term average of the different sinusoidal contributions tends to zero, when the time intervals exceed the largest signal period leads to Dimitriadis et al. (2009):

$$
\left\langle\Phi_{2, \mathbf{v}}[n(\mathbf{u})]\right\rangle \simeq \sum_{i} B_{i}^{2}\left(\eta_{i}^{T} \mathbf{v}\right)^{2}
$$


Finally, we obtain the following expression related to the ratio $R_{k}$ :

$$
R_{k} \simeq \frac{\sum_{i} B_{i}^{2}\left(\eta_{i}^{T} \mathbf{v}\right)^{2}}{A^{2}\left(\mathbf{w}^{T} \mathbf{v}\right)^{2}}
$$

Even adding a pre-filtering around the central frequency component of the AM-FM narrow band signal, approximation (29) remains not perfect due to the transient low pass terms. Indeed, the long term averaging still provides residual noisy sinusoidal terms. These remaining terms should be taken into account in presence of a local AM-FM low frequency narrow band signal. Thus, the ratio (30) remains correct i.e these transient terms could be neglected under the following hypotheses: (a) a relative higher SNR, which means that the instantaneous envelope of the signals exceeds the noisy amplitudes or b) the frequency of the AM-FM narrow band signal (i.e the norm || $\mathbf{w}||$ ) takes medium or high values. In our study, we focus on these assumptions. Moreover, as mentioned in Sect. 5, Eq. (26), applying the operator $\Phi_{B}$ to any local sinusoidal signal $s(\mathbf{u}) \simeq A \cos \left(\mathbf{w}^{T} \mathbf{u}\right)$ leads to $\Phi_{B}[s(\mathbf{u})]=A^{2}\|\mathbf{w}\|^{2}$. This yields the relation (31):

$$
R_{B} \simeq \frac{\sum_{i} B_{i}^{2}\left\|\eta_{i}\right\|^{2}}{A^{2}\|\mathbf{w}\|^{2}}
$$

To highlight the efficiency of these operators in presence of noise, we measure the rates (30) and (31) for different vectors w. For notational simplicity we restrict our theoretical discussion to $2 \mathrm{D}$ context.

\subsection{Comparison of operators in presence of noise in 2D and 3D context}

\subsubsection{Theoretical analysis}

We consider a single noisy 2D frequency component $\eta=(a b)^{T}$ associated with a random amplitude $B$, and a narrow band signal around the central frequency $\mathbf{w}=\left(w_{x} w_{y}\right)^{T}$. To compare the relative rates, we study separately the effects of each single noisy frequency component. The following relations (32) and (33) are compared:

$$
\begin{aligned}
& R_{k}=\frac{B^{2}\left(\boldsymbol{\eta}^{T} \mathbf{v}\right)^{2}}{A^{2}\left(\mathbf{w}^{T} \mathbf{v}\right)^{2}} \\
& R_{B}=\frac{B^{2}\|\boldsymbol{\eta}\|^{2}}{A^{2}\|\mathbf{w}\|^{2}}
\end{aligned}
$$

As mentioned in the Sect. 4.2, for our new operator, we have opted for the particular vector $\mathbf{v}=\left(\operatorname{sign}\left(w_{x}\right) \operatorname{sign}\left(w_{y}\right)\right)$. Finally, in a $2 \mathrm{D}$ context and for each noise frequency component, we compare the rates (34) and (35) for different noise and signal frequencies $\boldsymbol{\eta}$ and $\mathbf{w}$ :

$$
\begin{aligned}
R_{k} & =\frac{\left(a \operatorname{sign}\left(w_{x}\right)+b \operatorname{sign}\left(w_{y}\right)\right)^{2}}{\left(\left|w_{x}\right|+\left|w_{y}\right|\right)^{2}} \\
R_{B} & =\frac{a^{2}+b^{2}}{w_{x}^{2}+w_{y}^{2}}
\end{aligned}
$$

We highlight the frequencies $\mathbf{w}$ for which $R_{k}<R_{B}$ (resp. $R_{k} \geq R_{B}$ ). According to Eqs. (34) and (35) a simple calculus leads to the following relations: 
Fig. 5 Function

$f(x, y)=\frac{x y}{x^{2}+y^{2}}$

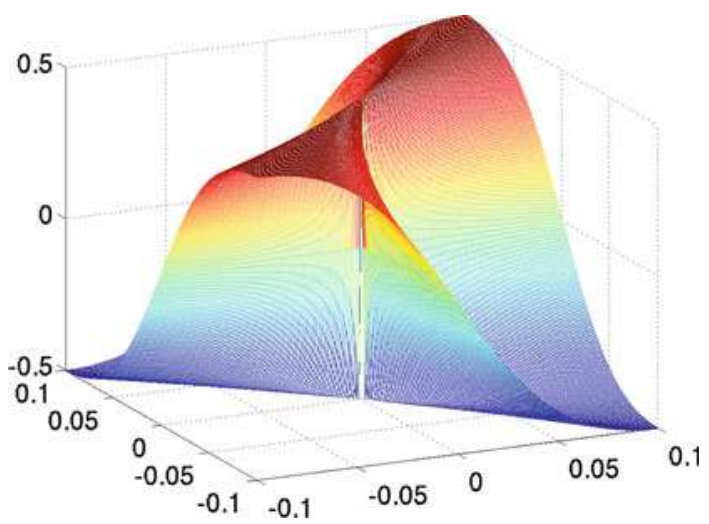

- If $\operatorname{sign}\left(w_{x} w_{y}\right)>0$ i.e., $w_{x}$ and $w_{y}$ own the same sign:

$$
R_{k}<R_{B} \Longleftrightarrow \frac{a b}{a^{2}+b^{2}}<\frac{w_{x} w_{y}}{w_{x}^{2}+w_{y}^{2}}
$$

- If $\operatorname{sign}\left(w_{x} w_{y}\right)<0$ i.e., $w_{x}$ and $w_{y}$ own different signs:

$$
R_{k}<R_{B} \Longleftrightarrow \frac{a b}{a^{2}+b^{2}}>\frac{w_{x} w_{y}}{w_{x}^{2}+w_{y}^{2}}
$$

It appears that the function $f(x, y)=\frac{x y}{x^{2}+y^{2}}$ provides a geometrical solution for which the rate of our new operator should be lower than the rate $R_{B}$ when the noisy component is modelled as a sum of sinusoids containing different random amplitudes and phases. This particular function is represented on Fig. 5.

Geometrically speaking, for a given point $\left(x_{0}, y_{0}\right)$ of the same sign (resp. different signs), which corresponds to a given frequency $\left(w_{x} w_{y}\right)$ the more the number of points $(x, y)$ i.e, noise vector $(a b)$ for which $f(x, y)<f\left(x_{0}, y_{0}\right)$ (respectively $\left.f(x, y)>f\left(x_{0}, y_{0}\right)\right)$ is higher, the more the rate $R_{k}$ will be better than $R_{B}$. According to Fig. 5 these properties are verified when $x_{0}$ i.e $w_{x}$ tends to be equal or the same order of magnitude than $y_{0}$ i.e, $w_{y}$. In the opposite way, the more $x_{0}$ and $y_{0}$ tends to be different, the higher $R_{B}$ will be from $R_{k}$. In other words, when the central frequency of the narrow band signal tends to be vertical or horizontal, the operator $\Phi_{B}$ should behave more efficiently than $\Phi_{2 \text {,sign(w). This }}$ property seems to be coherent with the definition of the operators: the operator $\Phi_{B}$ performs the mono-dimensional TK along the horizontal and the vertical axes, and so should be more sensitive to the diagonal orientations.

\subsubsection{Experimental validation}

To validate the assumptions made by the algorithmes in additive noise and on the frequency component values and to show the effectiveness of our model: (i) the rates $R_{B}$ and $R_{k}$ are computed using Eqs. (27-28) for different values and directions of the frequency vector $\mathbf{w}$ applying respectively the operators $\Phi_{B}$ and $\Phi_{2, \operatorname{sign}(\mathbf{w})}$ to a sinusoidal signal $s(\mathbf{u})=\cos \left(\mathbf{w}^{T} \mathbf{u}\right)$ and a pure additive noise component $n(\mathbf{u})$. These results are given in Table 9; (ii) Moreover, we have reported the global error rates $\varepsilon_{B}$ and $\varepsilon_{k}$ (resp. for the operators $\Phi_{B}$ and $\Phi_{2, \operatorname{sign}(\mathbf{w})}$ ) related to the estimation of the frequency components and according to different values and 
Table 9 2D sinusoid: ENR rates for $15 \%$ noisy pattern

\begin{tabular}{lllll}
\hline$w_{x}$ versus $w_{y}$ & $w_{x}=1 / 25$ & $w_{x}=1 / 15$ & $w_{x}=1 / 10$ & $w_{x}=1 / 5$ \\
\hline$w_{y}=0$ & $R_{k}=4.2 e-2$ & $R_{k}=1.6 e-2$ & $R_{k}=8.5 e-3$ & $R_{k}=4 e-3$ \\
& $R_{B}=2.6 e-2$ & $R_{B}=1 e-2$ & $R_{B}=5 e-3$ & $R_{B}=2.7 e-3$ \\
$w_{y}=1 / 100$ & $R_{k}=2.6 e-2$ & $R_{k}=1 . e-2$ & $R_{k}=6.9 e-3$ & $R_{k}=3.8 e-3$ \\
& $R_{B}=2.5 e-2$ & $R_{B}=1 e-2$ & $R_{B}=5.3 e-3$ & $R_{B}=2.6 e-3$ \\
$w_{y}=1 / 50$ & $R_{k}=1.8 e-2$ & $R_{k}=1 e-2$ & $R_{k}=6 e-3$ & $R_{k}=3.5 e-3$ \\
& $R_{B}=2.2 e-2$ & $R_{B}=1 e-2$ & $R_{B}=5.2 e-3$ & $R_{B}=2.6 e-3$ \\
$w_{y}=1 / 25$ & $R_{k}=1.1 e-2$ & $R_{k}=6.7 e-3$ & $R_{k}=4.6 e-3$ & $R_{k}=3.2 e-3$ \\
& $R_{B}=1.5 e-2$ & $R_{B}=8.2 e-3$ & $R_{B}=4.9 e-3$ & $R_{B}=2.5 e-3$ \\
$w_{y}=1 / 15$ & $R_{k}=6.8 e-3$ & $R_{k}=4.2 e-3$ & $R_{k}=3.4 e-3$ & $R_{k}=2.8 e-3$ \\
& $R_{B}=8.2 e-3$ & $R_{B}=6 e-3$ & $R_{B}=4.2 e-3$ & $R_{B}=2.7 e-3$ \\
$w_{y}=1 / 10$ & $R_{k}=4.6 e-3$ & $R_{k}=3.5 e-3$ & $R_{k}=3 e-3$ & $R_{k}=2.6 e-3$ \\
& $R_{B}=4.9 e-3$ & $R_{B}=4.2 e-3$ & $R_{B}=3.6 e-3$ & $R_{B}=2.7 e-3$ \\
$w_{y}=1 / 5$ & $R_{k}=3.1 e-3$ & $R_{k}=2.8 e-3$ & $R_{k}=2.6 e-3$ & $R_{k}=3.4 e-3$ \\
& $R_{B}=2.6 e-3$ & $R_{B}=2.8 e-3$ & $R_{B}=3 e-3$ & $R_{B}=4.2 e-3$
\end{tabular}

Comparison of $\Phi_{B}$ and $\Phi_{2, \operatorname{sign}(\mathbf{w})}$

directions of the frequency vector $\mathbf{w}$ of a noisy sinusoidal signal $s(\mathbf{u})=\cos \left(\mathbf{w}^{T} \mathbf{u}\right)+n(\mathbf{u})$. These results are given in Table 10. According to these results:

- The rates $R_{B}$ and $R_{k}$ of the TKEO depend on the central frequency vector: the higher the central frequency, the lower the rates will be. This corresponds to the given formulae of the respective rates: when the denominators tend to zero, the rates increase. Inversely, for higher central frequencies, the rates will decrease.

- For a given central frequency, the relative values of the ENR are strongly correlated with the relative quality of the estimation.

- The quality of the estimation rates $\varepsilon_{B}$ and $\varepsilon_{k}$ decreases for very low and very high frequencies w: actually, concerning the relatively low frequencies the rates $R_{B}$ and $R_{k}$ are higher. Concerning the very high frequency i.e $1 / 5$, all operators become sensitive to the derivatives.

In the $2 \mathrm{D}$ context, the results confirm our theoretical hypotheses: the more vertical (resp. horizontal) the carrier frequency of the signal tends to be, the better the operator $\Phi_{B}$ will behave relatively to $\Phi_{2, \operatorname{sign}(\mathbf{w})}$. Actually, for very low values of $w_{y}$ (i.e., $0,1 / 100$ ) the error rates (and also ENR rates) are better concerning the operator $\Phi_{B}$. When $w_{y} \simeq w_{x}$ with medium or higher values (i.e., 1/15, 1/25, 1/10,1/5) our operator tends to perform better, except when both frequency values are too different (i.e., $w_{x}=1 / 25 \mathrm{vs} w_{y}=1 / 5$ ): in that case, $\Phi_{B}$ behaves better.

\subsubsection{Extension to the $3 D$ context}

When processing 3D data, the difficulty in representing the geometrical solution makes the study more complex. However, it is possible to validate an extension of the previous assumption by an empirical approach. We have computed the ENR for different values and directions of the frequency components $w_{x}$ and $w_{y}$, fixing some values of the third component $w_{z}$. The $3 \mathrm{D}$ ENR values are given in Tables 11 and 13 for the respective values $w_{z}=0$ and $w_{z}=1 / 25$. 
Table 10 2D sinusoid: global frequency error for $15 \%$ noisy pattern

\begin{tabular}{lllll}
\hline$w_{x}$ versus $w_{y}$ & $w_{x}=1 / 25(\%)$ & $w_{x}=1 / 15(\%)$ & $w_{x}=1 / 10(\%)$ & $w_{x}=1 / 5(\%)$ \\
\hline$w_{y}=0$ & $\varepsilon_{k}=17.8$ & $\varepsilon_{k}=6.7$ & $\varepsilon_{k}=6.5$ & $\varepsilon_{k}=18.1$ \\
$w_{y}=1 / 100$ & $\varepsilon_{B}=17.5$ & $\varepsilon_{B}=6.1$ & $\varepsilon_{B}=5.9$ & $\varepsilon_{B}=17.8$ \\
& $\varepsilon_{k}=12.4$ & $\varepsilon_{k}=4.5$ & $\varepsilon_{k}=5.4$ & $\varepsilon_{k}=17.9$ \\
$w_{y}=1 / 50$ & $\varepsilon_{k}=6.3$ & $\varepsilon_{B}=4.6$ & $\varepsilon_{B}=5.3$ & $\varepsilon_{B}=17.8$ \\
& $\varepsilon_{B}=10.9$ & $\varepsilon_{k}=3.4$ & $\varepsilon_{k}=5.6$ & $\varepsilon_{k}=18$ \\
$w_{y}=1 / 25$ & $\varepsilon_{k}=3.9$ & $\varepsilon_{B}=4.25$ & $\varepsilon_{B}=5.5$ & $\varepsilon_{B}=17.9$ \\
& $\varepsilon_{B}=5.95$ & $\varepsilon_{k}=3.9$ & $\varepsilon_{k}=6.4$ & $\varepsilon_{k}=18.4$ \\
$w_{y}=1 / 15$ & $\varepsilon_{k}=3.9$ & $\varepsilon_{B}=4.3$ & $\varepsilon_{B}=6.4$ & $\varepsilon_{B}=18.2$ \\
& $\varepsilon_{B}=4.3$ & $\varepsilon_{k}=3.4$ & $\varepsilon_{B}=8.4$ & $\varepsilon_{k}=20.1$ \\
$w_{y}=1 / 10$ & $\varepsilon_{k}=6.4$ & $\varepsilon_{B}=5.4$ & $\varepsilon_{k}=11$ & $\varepsilon_{B}=20.1$ \\
& $\varepsilon_{B}=6.4$ & $\varepsilon_{k}=8.2$ & $\varepsilon_{B}=11.1$ & $\varepsilon_{k}=22.6$ \\
$w_{y}=1 / 5$ & $\varepsilon_{k}=18.4$ & $\varepsilon_{B}=8.4$ & $\varepsilon_{k}=22.6$ & $\varepsilon_{B}=22.6$ \\
& $\varepsilon_{B}=18.2$ & $\varepsilon_{k}=20.1$ & $\varepsilon_{B}=22.6$ & $\varepsilon_{k}=31.9$
\end{tabular}

Comparison of $\Phi_{B}$ and $\Phi_{2, \operatorname{sign}(\mathbf{w})}$

Moreover, the respective quality of the frequency estimation has been reported in Tables 12 and 14. With regard to the other components, let us define the small values of a frequency component as $\varepsilon$ (where $\varepsilon \simeq 0$ i.e, 0 or $1 / 100$ ), a "medium" value as $w_{x}, w_{y}$ or $w_{z}(1 / 50$ to $1 / 15$ cycles/pixel), the high value $1 / 10$ as $w_{x}^{+}, w_{y}^{+}$or $w_{z}^{+}$and the higher value $1 / 5$ as $w_{x}^{++}, w_{y}^{++}$or $w_{z}^{++}$. Again, according to these results, for a given central frequency, the relative values of the ENR are strongly correlated with the relative quality of the estimation. Also, the same observation as for 2D context concerning the level of ENR vs the central frequency. More precisely:

- The operator $\Phi_{B}$ performs better than $\Phi_{2, \operatorname{sign}(\mathbf{w})}$ (i.e, $R_{B} \leq R_{k}$ and $\varepsilon_{B} \leq \varepsilon_{k}$ ) under the following assumptions:

1. Two components of the frequency vector are small i.e, $\mathbf{w}=\left(w_{x}, \varepsilon, \varepsilon\right), \mathbf{w}=$ $\left(w_{x}^{+}, \varepsilon, \varepsilon\right)$ or $\mathbf{w}=\left(w_{x}^{++}, \varepsilon, \varepsilon\right)$. In that case the frequency vector is almost monodimensional;

2. All values are different $\mathbf{w}=\left(w_{x}^{+}, w_{y}, \varepsilon\right), \mathbf{w}=\left(w_{x}^{+}, \varepsilon, w_{z}\right), \mathbf{w}=\left(w_{x}^{++}, w_{y}, \varepsilon\right)$ or $\mathbf{w}=\left(w_{x}^{++}, \varepsilon, w_{z}\right)$;

3. One frequency component is very high and two components are medium i.e, $\mathbf{w}=$ $\left(w_{x}^{++}, w_{y}, w_{z}\right)$;

4. Two frequency components own higher value vs one small value for one component i.e, $\mathbf{w}=\left(w_{x}^{+}, w_{y}^{+}, \varepsilon\right)$ or $\mathbf{w}=\left(w_{x}^{++}, w_{y}^{++}, \varepsilon\right) ;$

- The operator $\Phi_{2, \operatorname{sign}(\mathbf{w})}$ performs better than $\Phi_{B}$ (i.e, $R_{k} \leq R_{B}$ and $\varepsilon_{k} \leq \varepsilon_{B}$ ) under the following assumptions:

1. Two components are "medium" and one component is small i.e, $\mathbf{w}=\left(w_{x}, w_{y}, \varepsilon\right)$ or $\mathbf{w}=\left(w_{x}, \varepsilon, w_{z}\right)$

2. All components own similar order of medium values $\mathbf{w}=\left(w_{x}, w_{y}, w_{z}\right)$;

3. One component is high vs two medium values i.e, $\mathbf{w}=\left(w_{x}^{+}, w_{y}, w_{z}\right)$; 
Table 11 3D sinusoid: ENR rates for $15 \%$ noisy pattern and $w_{z}=0$

\begin{tabular}{lllll}
\hline$w_{x}$ versus $w_{y}$ & $w_{x}=1 / 25$ & $w_{x}=1 / 15$ & $w_{x}=1 / 10$ & $w_{x}=1 / 5$ \\
\hline$w_{y}=0$ & $R_{k}=6.9 e-2$ & $R_{k}=2.5 e-2$ & $R_{k}=1.2 e-2$ & $R_{k}=4.7 e-3$ \\
$w_{y}=1 / 100$ & $R_{B}=3.4 e-2$ & $R_{B}=1.1 e-2$ & $R_{B}=5.2 e-3$ & $R_{B}=2.1 e-3$ \\
& $R_{k}=4.2 e-2$ & $R_{k}=1.9 e-2$ & $R_{k}=1 e-2$ & $R_{k}=4.2 e-3$ \\
$w_{y}=1 / 50$ & $R_{B}=3.1 e-2$ & $R_{B}=1.3 e-2$ & $R_{B}=5.3 e-3$ & $R_{B}=2 e-3$ \\
& $R_{k}=2.5 e-2$ & $R_{k}=1.3 e-2$ & $R_{k}=8.4 e-3$ & $R_{k}=3.9 e-3$ \\
$w_{y}=1 / 25$ & $R_{B}=2.8 e-2$ & $R_{B}=1.2 e-2$ & $R_{B}=5.1 e-3$ & $R_{B}=2 e-3$ \\
& $R_{k}=1.5 e-2$ & $R_{k}=8.2 e-3$ & $R_{k}=6.3 e-3$ & $R_{k}=3.4 e-3$ \\
$w_{y}=1 / 15$ & $R_{B}=1.7 e-2$ & $R_{B}=1 e-2$ & $R_{B}=4.6 e-3$ & $R_{B}=2 e-3$ \\
& $R_{k}=8.2 e-3$ & $R_{k}=6 e-3$ & $R_{k}=4.7 e-3$ & $R_{k}=2.9 e-3$ \\
$w_{y}=1 / 10$ & $R_{B}=1 e-2$ & $R_{B}=6.7 e-3$ & $R_{B}=4 e-3$ & $R_{B}=2 e-3$ \\
& $R_{k}=6.3 e-3$ & $R_{k}=4.7 e-3$ & $R_{k}=3.7 e-3$ & $R_{k}=2.7 e-3$ \\
$w_{y}=1 / 5$ & $R_{B}=4.7 e-3$ & $R_{B}=3.9 e-3$ & $R_{B}=3.2 e-3$ & $R_{B}=2.1 e-3$ \\
& $R_{k}=3.4 e-3$ & $R_{k}=2.9 e-3$ & $R_{k}=2.7 e-3$ & $R_{k}=2.6 e-3$ \\
& $R_{B}=2 e-3$ & $R_{B}=2 e-3$ & $R_{B}=2.1 e-3$ & $R_{B}=2.3 e-3$
\end{tabular}

Comparison of $\Phi_{B}$ and $\Phi_{2, \operatorname{sign}(\mathbf{w})}$

Table 12 3D sinusoid: global frequency error for $15 \%$ noisy pattern and $w_{z}=0$

\begin{tabular}{lllll}
\hline$w_{x}$ versus $w_{y}$ & $w_{x}=1 / 25(\%)$ & $w_{x}=1 / 15(\%)$ & $w_{x}=1 / 10(\%)$ & $w_{x}=1 / 5(\%)$ \\
\hline$w_{y}=0$ & $\varepsilon_{k}=24.3$ & $\varepsilon_{k}=9.6$ & $\varepsilon_{k}=6.2$ & $\varepsilon_{k}=12.9$ \\
$w_{y}=1 / 100$ & $\varepsilon_{B}=24.1$ & $\varepsilon_{B}=8.1$ & $\varepsilon_{B}=4.9$ & $\varepsilon_{B}=12.2$ \\
& $\varepsilon_{k}=21.3$ & $\varepsilon_{k}=7.25$ & $\varepsilon_{k}=5.1$ & $\varepsilon_{k}=12.7$ \\
$w_{y}=1 / 50$ & $\varepsilon_{B}=18.2$ & $\varepsilon_{B}=6.9$ & $\varepsilon_{B}=4.4$ & $\varepsilon_{B}=12.3$ \\
& $\varepsilon_{k}=12.9$ & $\varepsilon_{k}=6.2$ & $\varepsilon_{k}=4.8$ & $\varepsilon_{k}=12.7$ \\
$w_{y}=1 / 25$ & $\varepsilon_{B}=17.3$ & $\varepsilon_{B}=6.4$ & $\varepsilon_{B}=4.5$ & $\varepsilon_{B}=12.4$ \\
$w_{y}=1 / 15$ & $\varepsilon_{k}=7.5$ & $\varepsilon_{k}=4.8$ & $\varepsilon_{k}=5.0$ & $\varepsilon_{k}=13.3$ \\
& $\varepsilon_{B}=9.9$ & $\varepsilon_{B}=5.4$ & $\varepsilon_{B}=4.9$ & $\varepsilon_{B}=13.0$ \\
$w_{y}=1 / 10$ & $\varepsilon_{k}=4.8$ & $\varepsilon_{k}=4.6$ & $\varepsilon_{B}=5.9$ & $\varepsilon_{k}=14.5$ \\
& $\varepsilon_{B}=5.4$ & $\varepsilon_{B}=4.9$ & $\varepsilon_{k}=8.1$ & $\varepsilon_{B}=14.3$ \\
$w_{y}=1 / 5$ & $\varepsilon_{k}=5.0$ & $\varepsilon_{k}=6.0$ & $\varepsilon_{B}=7.8$ & $\varepsilon_{k}=16.7$ \\
& $\varepsilon_{B}=4.9$ & $\varepsilon_{B}=6.0$ & $\varepsilon_{k}=16.7$ & $\varepsilon_{B}=16.6$ \\
\hline
\end{tabular}

Comparison of $\Phi_{B}$ and $\Phi_{2, \operatorname{sign}(\mathbf{w})}$

4. Two components are high vs medium value i.e, $\mathbf{w}=\left(w_{x}^{+}, w_{y}^{+}, w_{z}\right)$ or $\mathbf{w}=$ $\left(w_{x}^{++}, w_{y}^{++}, w_{z}\right)$

Moreover, we have performed a simulation when $\mathbf{w}=\left(w_{x}^{+}, w_{y}^{+}, w_{z}^{+}\right)$and $\mathbf{w}=$ $\left(w_{x}^{++}, w_{y}^{++}, w_{z}^{++}\right)$. The result provides: $R_{k} \leq R_{B}$ and also $\varepsilon_{k} \leq \varepsilon_{B}$. 
Table 13 3D sinusoid: ENR rates for $15 \%$ noisy pattern and $w_{z}=1 / 25$

\begin{tabular}{|c|c|c|c|c|c|c|c|}
\hline$w_{x}$ versus $w_{y}$ & $w_{x}=0$ & $w_{x}=1 / 100$ & $w_{x}=1 / 50$ & $w_{x}=1 / 25$ & $w_{x}=1 / 15$ & $w_{x}=1 / 10$ & $w_{x}=1 / 5$ \\
\hline \multirow[t]{2}{*}{$w_{y}=0$} & $R_{k}=6.9 e-2$ & $R_{k}=4.2 e-2$ & $R_{k}=2.5 e-2$ & $R_{k}=1.5 e-2 \mathrm{~h}$ & $R_{k}=8.2 e-3$ & $R_{k}=6.3 e-3$ & $R_{k}=3.4 e-3$ \\
\hline & $R_{B}=3.4 e-2$ & $R_{B}=3.1 e-2$ & $R_{B}=2.8 e-2$ & $R_{B}=1.7 e-2$ & $R_{B}=1 e-2$ & $R_{B}=4.7 e-3$ & $R_{B}=2 e-3$ \\
\hline \multirow[t]{2}{*}{$w_{y}=1 / 100$} & $R_{k}=4.2 e-2$ & $R_{k}=2.9 e-2$ & $R_{k}=2.2 e-2$ & $R_{k}=1.3 e-2$ & $R_{k}=8.1 e-3$ & $R_{k}=5.5 e-3$ & $R_{k}=3.1 e-3$ \\
\hline & $R_{B}=3.1 e-2$ & $R_{B}=2.6 e-2$ & $R_{B}=2.2 e-2$ & $R_{B}=1.4 e-2$ & $R_{B}=8.3 e-3$ & $R_{B}=4.6 e-3$ & $R_{B}=2 e-3$ \\
\hline \multirow[t]{2}{*}{$w_{y}=1 / 50$} & $R_{k}=2.5 e-2$ & $R_{k}=2.2 e-2$ & $R_{k}=1.7 e-2$ & $R_{k}=1.1 e-2$ & $R_{k}=7.1 e-3$ & $R_{k}=4.5 e-3$ & $R_{k}=3 e-3$ \\
\hline & $R_{B}=2.8 e-2$ & $R_{B}=2.2 e-2$ & $R_{B}=2 e-2$ & $R_{B}=1.4 e-2$ & $R_{B}=7.8 e-3$ & $R_{B}=5 e-3$ & $R_{B}=2 e-3$ \\
\hline \multirow[t]{2}{*}{$w_{y}=1 / 25$} & $R_{k}=1.5 e-2$ & $R_{k}=1.3 e-2$ & $R_{k}=1.1 e-2$ & $R_{k}=7.7 e-3$ & $R_{k}=5.4 e-3$ & $R_{k}=3.9 e-3$ & $R_{k}=2.6 e-3$ \\
\hline & $R_{B}=1.7 e-2$ & $R_{B}=1.4 e-2$ & $R_{B}=1.4 e-2$ & $R_{B}=1 e-2$ & $R_{B}=6.7 e-3$ & $R_{B}=4.2 e-3$ & $R_{B}=2 e-3$ \\
\hline \multirow[t]{2}{*}{$w_{y}=1 / 15$} & $R_{k}=8.2 e-3$ & $R_{k}=8.1 e-3$ & $R_{k}=7.1 e-3$ & $R_{k}=5.4 e-3$ & $R_{k}=4.1 e-3$ & $R_{k}=3.1 e-3$ & $R_{k}=2.3 e-3$ \\
\hline & $R_{B}=1 e-2$ & $R_{B}=8.1 e-3$ & $R_{B}=7.8 e-3$ & $R_{B}=6.7 e-3$ & $R_{B}=5.1 e-3$ & $R_{B}=3.7 e-3$ & $R_{B}=2 e-3$ \\
\hline \multirow[t]{2}{*}{$w_{y}=1 / 10$} & $R_{k}=6.3 e-3$ & $R_{k}=5.5 e-3$ & $R_{k}=5 e-3$ & $R_{k}=3.9 e-3$ & $R_{k}=3.1 e-3$ & $R_{k}=2.6 e-3$ & $R_{k}=2.1 e-3$ \\
\hline & $R_{B}=4.7 e-3$ & $R_{B}=4.6 e-3$ & $R_{B}=4.5 e-3$ & $R_{B}=4.2 e-3$ & $R_{B}=3.7 e-3$ & $R_{B}=3 e-3$ & $R_{B}=2 e-3$ \\
\hline \multirow[t]{2}{*}{$w_{y}=1 / 5$} & $R_{k}=3.4 e-3$ & $R_{k}=3.1 e-3$ & $R_{k}=3 e-3$ & $R_{k}=2.6 e-3$ & $R_{k}=2.3 e-3$ & $R_{k}=2.1 e-3$ & $R_{k}=2.2 e-3$ \\
\hline & $R_{B}=2 e-3$ & $R_{B}=2 e-3$ & $R_{B}=2 e-3$ & $R_{B}=2 e-3$ & $R_{B}=2 e-3$ & $R_{B}=2 e-3$ & $R_{B}=2.3 e-3$ \\
\hline
\end{tabular}

Comparison of $\Phi_{B}$ and $\Phi_{2, \operatorname{sign}(\mathbf{w})}$ 
Table 14 3D sinusoid: global frequency error for $15 \%$ noisy pattern and $w_{z}=1 / 25$

\begin{tabular}{llllllll}
\hline$w_{x}$ versus $w_{y}$ & $w_{x}=0$ & $w_{x}=1 / 100$ & $w_{x}=1 / 50$ & $w_{x}=1 / 25$ & $w_{x}=1 / 15$ & $w_{x}=1 / 10$ & $w_{x}=1 / 5$ \\
\hline$w_{y}=0$ & $\varepsilon_{k}=24.3$ & $\varepsilon_{k}=21.3$ & $\varepsilon_{k}=12.9$ & $\varepsilon_{k}=7.5$ & $\varepsilon_{k}=4.8$ & $\varepsilon_{k}=5.0$ & $\varepsilon_{k}=13.3$ \\
& $\varepsilon_{B}=24.0$ & $\varepsilon_{B}=18.3$ & $\varepsilon_{B}=17.3$ & $\varepsilon_{B}=9.9$ & $\varepsilon_{B}=5.4$ & $\varepsilon_{B}=4.9$ & $\varepsilon_{B}=13.0$ \\
$w_{y}=1 / 100$ & $\varepsilon_{k}=21.3$ & $\varepsilon_{k}=18.6$ & $\varepsilon_{k}=8.0$ & $\varepsilon_{k}=4.6$ & $\varepsilon_{k}=3.3$ & $\varepsilon_{k}=4.5$ & $\varepsilon_{k}=13.1$ \\
& $\varepsilon_{B}=18.3$ & $\varepsilon_{B}=14.2$ & $\varepsilon_{B}=12.9$ & $\varepsilon_{B}=8.2$ & $\varepsilon_{B}=4.4$ & $\varepsilon_{B}=4.4$ & $\varepsilon_{B}=13.1$ \\
$w_{y}=1 / 50$ & $\varepsilon_{k}=12.9$ & $\varepsilon_{k}=8.0$ & $\varepsilon_{k}=5.6$ & $\varepsilon_{k}=3.8$ & $\varepsilon_{k}=3.3$ & $\varepsilon_{k}=4.6$ & $\varepsilon_{k}=13.2$ \\
& $\varepsilon_{B}=17.3$ & $\varepsilon_{B}=12.9$ & $\varepsilon_{B}=12.0$ & $\varepsilon_{B}=7.2$ & $\varepsilon_{B}=4.3$ & $\varepsilon_{B}=4.7$ & $\varepsilon_{B}=13.2$ \\
$w_{y}=1 / 25$ & $\varepsilon_{k}=7.5$ & $\varepsilon_{k}=4.6$ & $\varepsilon_{k}=3.8$ & $\varepsilon_{k}=3.3$ & $\varepsilon_{k}=3.6$ & $\varepsilon_{k}=5.2$ & $\varepsilon_{k}=13.7$ \\
& $\varepsilon_{B}=9.9$ & $\varepsilon_{B}=8.2$ & $\varepsilon_{B}=7.2$ & $\varepsilon_{B}=5.3$ & $\varepsilon_{B}=4.2$ & $\varepsilon_{B}=5.2$ & $\varepsilon_{B}=13.5$ \\
$w_{y}=1 / 15$ & $\varepsilon_{k}=4.8$ & $\varepsilon_{k}=3.3$ & $\varepsilon_{k}=3.3$ & $\varepsilon_{k}=3.6$ & $\varepsilon_{k}=4.5$ & $\varepsilon_{k}=6.3$ & $\varepsilon_{k}=15.0$ \\
& $\varepsilon_{B}=5.4$ & $\varepsilon_{B}=4.4$ & $\varepsilon_{B}=4.3$ & $\varepsilon_{B}=4.2$ & $\varepsilon_{B}=4.8$ & $\varepsilon_{B}=6.4$ & $\varepsilon_{B}=15.0$ \\
$w_{y}=1 / 10$ & $\varepsilon_{k}=5.0$ & $\varepsilon_{k}=4.5$ & $\varepsilon_{k}=4.6$ & $\varepsilon_{k}=5.2$ & $\varepsilon_{k}=6.3$ & $\varepsilon_{k}=8.4$ & $\varepsilon_{k}=17.2$ \\
& $\varepsilon_{B}=4.9$ & $\varepsilon_{B}=4.4$ & $\varepsilon_{B}=4.7$ & $\varepsilon_{B}=5.2$ & $\varepsilon_{B}=6.4$ & $\varepsilon_{B}=8.5$ & $\varepsilon_{B}=17.2$ \\
$w_{y}=1 / 5$ & $\varepsilon_{k}=13.3$ & $\varepsilon_{k}=13.1$ & $\varepsilon_{k}=13.2$ & $\varepsilon_{k}=13.7$ & $\varepsilon_{k}=15.0$ & $\varepsilon_{k}=17.2$ & $\varepsilon_{k}=25.6$ \\
& $\varepsilon_{B}=13.0$ & $\varepsilon_{B}=13.1$ & $\varepsilon_{B}=13.2$ & $\varepsilon_{B}=13.5$ & $\varepsilon_{B}=15.0$ & $\varepsilon_{B}=17.2$ & $\varepsilon_{B}=25.6$ \\
\hline
\end{tabular}

Comparison of $\Phi_{B}$ and $\Phi_{2, \operatorname{sign}(\mathbf{w})}$

Finally, although it is difficult to establish an exact mathematical rule which indicates the respective performances of the algorithms, the operator $\Phi_{B}$ behaves better than $\Phi_{2, \operatorname{sign}(\mathbf{w})}$ when the frequency components are more dispersed or extreme.

\section{Conclusion}

In this paper, we have introduced a generalization of TKEO and higher order differential operators to multi-dimensional signals using higher order gradients combined with Kronecker product. In particular we have highlighted the link between these tensors and any directional derivatives along a vector, which allows us to construct a large class of functions. An appropriate choice of the directional vector leads to operators which are proportional to a quadratic norm of the frequency. An important aspect of our algorithm is the ability to demodulate local AM-FM signals. We have been introduced a new scalar operator corresponding to a directional derivative along the vector containing the "sign" of the frequency vector. A comparison with some multi-dimensional energy separation algorithms shows the competitiveness and robustness of our approach to track the instantaneous envelope and frequency components. The theoretical comparison between the most used demodulation methods has been proposed, which leads to a geometrical solution, validated by an empirical approach. Finally, as future work, we plan to compare our approach to recent numerical improved version of $2 \mathrm{D}$ energy operator and related 2D AM-FM demodulation algorithm that use regularization version of the 2D TKEO (Kokkinos et al. 2009).

Acknowledgments The authors would like to thank the Editor and reviewers for their valuable comments and suggestions which improved the quality of the paper. We thank Ms Claire Bloomfield for correction of the English. 


\section{A Relation between higher order gradients and directional derivatives using Kronecker product}

The derivative of a product $\mathbf{A B}$ yields:

$$
\begin{aligned}
\frac{d}{d \mathbf{u}}(\mathbf{A B}) & =\left(\frac{\partial(\mathbf{A} \mathbf{B})}{\partial x_{1}} \ldots \frac{\partial(\mathbf{A B})}{\partial x_{n}}\right) \\
& =\left(\frac{\partial \mathbf{A}}{\partial x_{1}} \mathbf{B}+\mathbf{A} \frac{\partial \mathbf{B}}{\partial x_{1}} \ldots \frac{\partial \mathbf{A}}{\partial x_{n}} \mathbf{B}+\mathbf{A} \frac{\partial \mathbf{B}}{\partial x_{n}}\right) \\
& =\left(\frac{\partial \mathbf{A}}{\partial x_{1}} \ldots \frac{\partial \mathbf{A}}{\partial x_{n}}\right)\left(\begin{array}{cccc}
\mathbf{B} & \mathbf{0} & \ldots & \mathbf{0} \\
\mathbf{0} & \mathbf{B} & \ldots & \mathbf{0} \\
\mathbf{0} & \ldots & \mathbf{0} & \mathbf{B}
\end{array}\right) \\
& +\mathbf{A}\left(\frac{\partial \mathbf{B}}{\partial x_{1}} \ldots \frac{\partial \mathbf{B}}{\partial x_{n}}\right) \\
& =\frac{d \mathbf{A}}{d \mathbf{u}}\left(\mathbf{I}_{\mathbf{n}} \otimes \mathbf{B}\right)+\mathbf{A} \frac{d \mathbf{B}}{d \mathbf{u}}
\end{aligned}
$$

where $\mathbf{I}_{\mathbf{n}}$ represents the identity matrix $\mathbf{n} \times \mathbf{n}$. A general result dealing with derivatives according to a matrix (instead of a vector) can be found in Brewer (1978). In particular, we derive the following properties, where $\mathbf{v}$ and $\mathbf{w}$ represent any constant vectors, provided that the dimensions are compatible:

$$
\frac{d}{d \mathbf{u}}(\mathbf{v A w})=\mathbf{v} \frac{d \mathbf{A}}{d \mathbf{u}}\left(\mathbf{I}_{\mathbf{n}} \otimes \mathbf{w}\right)
$$

Moreover, a property of the Kronecker product (Moon and Stirling 2000) yields:

$$
(\mathbf{A} \otimes \mathbf{B})(\mathbf{C} \otimes \mathbf{D})=(\mathbf{A C}) \otimes(\mathbf{B D})
$$

We deduce, provided that the dimensions are compatible:

$$
\begin{aligned}
& \left(\mathbf{A}_{\mathbf{1}} \otimes \mathbf{B}_{\mathbf{1}}\right)\left(\mathbf{A}_{\mathbf{2}} \otimes \mathbf{B}_{\mathbf{2}}\right)\left(\mathbf{A}_{\mathbf{3}} \otimes \mathbf{B}_{3}\right) \\
= & \left(\left(\mathbf{A}_{\mathbf{1}} \mathbf{A}_{2}\right) \otimes\left(\mathbf{B}_{\mathbf{1}} \mathbf{B}_{\mathbf{2}}\right)\right)\left(\mathbf{A}_{\mathbf{3}} \otimes \mathbf{B}_{3}\right) \\
= & \left(\mathbf{A}_{\mathbf{1}} \mathbf{A}_{\mathbf{2}} \mathbf{A}_{3}\right) \otimes\left(\mathbf{B}_{\mathbf{1}} \mathbf{B}_{\mathbf{2}} \mathbf{B}_{3}\right)
\end{aligned}
$$

From (40) it follows:

$$
\left(\mathbf{I}_{\mathbf{n}} \otimes \mathbf{v}\right) \mathbf{v}=\left(\mathbf{I}_{\mathbf{n}} \otimes \mathbf{v}\right)\left(\mathbf{v} \otimes \mathbf{I}_{\mathbf{1}}\right)=\left(\mathbf{I}_{\mathbf{n}} \mathbf{v}\right) \otimes\left(\mathbf{v} \mathbf{I}_{\mathbf{1}}\right)=\mathbf{v} \otimes \mathbf{v}
$$

Moreover, we have the following relation:

$$
\begin{aligned}
\left(\mathbf{I}_{\mathbf{n}} \otimes \mathbf{v} \otimes \mathbf{v}\right) \mathbf{v} & =\left(\mathbf{A}_{\mathbf{n}} \otimes \mathbf{v}\right)\left(\mathbf{v} \otimes \mathbf{I}_{\mathbf{1}}\right) \\
& =\left(\mathbf{A}_{\mathbf{n}} \mathbf{v}\right) \otimes\left(\mathbf{v} \mathbf{I}_{\mathbf{1}}\right)
\end{aligned}
$$

where $\mathbf{A}_{\mathbf{n}}=\mathbf{I}_{\mathbf{n}} \otimes \mathbf{v}$. From (43) it follows $\mathbf{A}_{\mathbf{n}} \mathbf{v}=\mathbf{v} \otimes \mathbf{v}$. Finally (44) provides:

$$
\left(\mathbf{I}_{\mathbf{n}} \otimes \mathbf{v} \otimes \mathbf{v}\right) \mathbf{v}=\mathbf{v} \otimes \mathbf{v} \otimes \mathbf{v}
$$

Unsing $\bigotimes_{k} \mathbf{v}=\mathbf{v} \otimes \cdots \otimes \mathbf{v}$, we deduce by recurrence:

$$
\left(\mathbf{I}_{\mathbf{n}} \otimes \bigotimes_{k} \mathbf{v}\right) \mathbf{v}=\bigotimes_{k+1} \mathbf{v}
$$


Let us now express the relation between the third order gradient and the third order directional derivative:

$$
\begin{aligned}
\frac{\partial^{3} s}{\partial \mathbf{v}^{3}}(\mathbf{u}) & =\frac{\partial}{\partial \mathbf{v}}\left[\frac{\partial^{2} s}{\partial \mathbf{v}^{2}}(\mathbf{u})\right]^{T}=\frac{d}{d \mathbf{u}}\left[\frac{\partial^{2} s}{\partial \mathbf{v}^{2}}(\mathbf{u})\right]^{T} \mathbf{v} \\
& =\frac{d}{d \mathbf{u}}\left[\mathbf{v}^{T} \frac{d^{2} s}{d \mathbf{u}^{2}} \mathbf{v}\right]^{T} \mathbf{v} \\
& =\frac{d}{d \mathbf{u}}\left[\mathbf{v}^{T}\left(\frac{d^{2} s}{d \mathbf{u}^{2}}\right)^{T} \mathbf{v}\right] \mathbf{v}
\end{aligned}
$$

Using Eq. (39), relation (47) can be written:

$$
\frac{d}{d \mathbf{u}}\left[\mathbf{v}^{T}\left(\frac{d^{2} s}{d \mathbf{u}^{2}}\right)^{T} \mathbf{v}\right] \mathbf{v}=\mathbf{v}^{T} \frac{d^{3} s}{d \mathbf{u}^{3}}\left(\mathbf{I}_{\mathbf{n}} \otimes \mathbf{v}\right) \mathbf{v}
$$

Finally (46) and (48) where $k=1$ give:

$$
\frac{\partial^{3} s}{\partial \mathbf{v}^{3}}(\mathbf{u})=\mathbf{v}^{T} \frac{d^{3} s}{d \mathbf{u}^{3}}(\mathbf{v} \otimes \mathbf{v})
$$

The dimensions are compatible. Let us now compute the fourth order derivative:

$$
\begin{aligned}
\frac{\partial^{4} s}{\partial \mathbf{v}^{4}}(\mathbf{u}) & =\frac{\partial}{\partial \mathbf{v}}\left[\frac{\partial^{3} s}{\partial \mathbf{v}^{3}}(\mathbf{u})\right]^{T} \\
& =\frac{d}{d \mathbf{u}}\left[\frac{\partial^{3} s}{\partial \mathbf{v}^{3}}(\mathbf{u})\right]^{T} \mathbf{v} \\
& =\frac{d}{d \mathbf{u}}\left[\mathbf{v}^{T} \frac{d^{3} s}{d \mathbf{u}^{3}} \mathbf{w}\right]^{T} \mathbf{v} \\
& =\frac{d}{d \mathbf{u}}\left[\mathbf{w}^{T}\left(\frac{d^{3} s}{d \mathbf{u}^{3}}\right)^{T} \mathbf{v}\right] \mathbf{v} \\
& =\mathbf{w}^{T} \frac{d^{4} s}{d \mathbf{u}^{4}}\left(\mathbf{I}_{\mathbf{n}} \otimes \mathbf{v}\right) \mathbf{v}
\end{aligned}
$$

where $\mathbf{w}=\mathbf{v} \otimes \mathbf{v}$. According to (46) and the property $(\mathbf{v} \otimes \mathbf{v})^{T}=\mathbf{v}^{\mathbf{T}} \otimes \mathbf{v}^{\mathbf{T}}$ Eq. (50) yields:

$$
\frac{\partial^{4} s}{\partial \mathbf{v}^{4}}(\mathbf{u})=\left(\mathbf{v}^{\mathbf{T}} \otimes \mathbf{v}^{\mathbf{T}}\right) \frac{d^{4} s}{d \mathbf{u}^{4}}(\mathbf{v} \otimes \mathbf{v})
$$

Equation (46) yields the following rules by recurrence:

$$
\begin{aligned}
& \text { for an even order } k=2 p \text { : } \\
& \frac{\partial^{k} s}{\partial \mathbf{v}^{k}}(\mathbf{u})=\left(\bigotimes_{p} \mathbf{v}^{\mathbf{T}}\right) \frac{d^{k} s}{d \mathbf{u}^{k}} \bigotimes_{p} \mathbf{v} \\
& \text { for an odd order } k=2 p+1 \text { : } \\
& \frac{\partial^{k} s}{\partial \mathbf{v}^{k}}(\mathbf{u})=\left(\bigotimes_{p} \mathbf{v}^{\mathbf{T}}\right) \frac{d^{k} s}{d \mathbf{u}^{k}} \bigotimes_{p+1} \mathbf{v}
\end{aligned}
$$




\section{B Proof the Proposition 1}

Let us multiply the tensors by a $p+q=m+l+1$ th Kronecker product of the $\mathbf{n} \times \mathbf{1}$ vector v. Applying Eqs. (41) and (52) yields:

$$
\begin{aligned}
& \left(\bigotimes_{p+q} \mathbf{v}^{\mathbf{T}}\right)\left(\frac{d^{2 p} s}{d \mathbf{u}^{2 p}} \otimes\left(\frac{d^{2 q} s}{d \mathbf{u}^{2 q}}\right)^{T}\right) \bigotimes_{p+q} \mathbf{v} \\
= & \left(\left(\bigotimes_{p} \mathbf{v}^{\mathbf{T}}\right) \otimes\left(\bigotimes_{q} \mathbf{v}^{\mathbf{T}}\right)\right)\left(\frac{d^{2 p} s}{d \mathbf{u}^{2 p}} \otimes\left(\frac{d^{2 q} s}{d \mathbf{u}^{2 q}}\right)^{T}\right)\left(\bigotimes_{p} \mathbf{v} \otimes \bigotimes_{q} \mathbf{v}\right) \\
= & \left(\left(\bigotimes_{p} \mathbf{v}^{\mathbf{T}}\right) \frac{d^{2 p} s}{d \mathbf{u}^{2 p}} \bigotimes_{p} \mathbf{v}\right)\left(\left(\bigotimes_{q} \mathbf{v}^{\mathbf{T}}\right)\left(\frac{d^{2 q} s}{d \mathbf{u}^{2 q}}\right)^{T} \bigotimes_{q} \mathbf{v}\right) \\
= & \frac{\partial^{2 p} s}{\partial \mathbf{v}^{2 p}}\left(\frac{\partial^{2 q} s}{\partial \mathbf{v}^{2 q}}\right)^{T}
\end{aligned}
$$

In the same manner, applying Eqs. (41) and (53) yields:

$$
\begin{aligned}
& \left(\bigotimes_{m+l+1} \mathbf{v}^{\mathbf{T}}\right)\left(\frac{d^{2 m+1} s}{d \mathbf{u}^{2 m+1}} \otimes\left(\frac{d^{2 l+1} s}{d \mathbf{u}^{2 l+1}}\right)^{T}\right)\left(\bigotimes_{m+l+1} \mathbf{v}\right) \\
= & \left.\left(\bigotimes_{m} \mathbf{v}^{\mathbf{T}}\right) \frac{d^{2 m+1} s}{d \mathbf{u}^{2 m+1}} \bigotimes_{m+1} \mathbf{v}\right)\left(\bigotimes_{l+1} \mathbf{v}^{\mathbf{T}}\left(\frac{d^{2 l+1} s}{d \mathbf{u}^{2 l+1}}\right)^{T} \bigotimes_{l} \mathbf{v}\right) \\
= & \frac{\partial^{2 m+1} s}{\partial \mathbf{v}^{2 m+1}}\left(\frac{\partial^{2 l+1} s}{\partial \mathbf{v}^{2 l+1}}\right)^{T}
\end{aligned}
$$

which completes the proof.

\section{Computation of the error}

Let us consider the $n$ dimensional TKEO which yields the following expression:

$$
\Phi_{2, \mathbf{v}}[s(\mathbf{u})]=\mathbf{v}^{T} \cdot \Psi_{2}[s(\mathbf{u})] \cdot \mathbf{v}=\frac{\partial s}{\partial \mathbf{v}} \frac{\partial s}{\partial \mathbf{v}}-s \frac{\partial^{2} s}{\partial \mathbf{v}^{2}}
$$

Consider a general AM-FM signal:

$$
s(\mathbf{u})=A(\mathbf{u}) \cos (\phi(\mathbf{u}))=A(\mathbf{u}) B(\mathbf{u})
$$

We now provide the conditions under which the approximation (54) remains true:

$$
\Phi_{2, \mathbf{v}}[s(\mathbf{u})] \simeq A(\mathbf{u})^{2}\left(\frac{\partial \phi}{\partial \mathbf{v}}(\mathbf{u})\right)^{2}=A(\mathbf{u})^{2}\left(\mathbf{w}(\mathbf{u})^{T} \mathbf{v}\right)^{2}
$$

The frequency vector is given by:

$$
\mathbf{w}(\mathbf{u})=\left(w_{1}(\mathbf{u}), w_{2}(\mathbf{u}), \ldots, w_{n}(\mathbf{u})\right)^{T}=\frac{d \phi}{d \mathbf{u}}
$$


where

$$
w_{i}(\mathbf{u})=\frac{\partial \phi}{\partial x_{i}}(\mathbf{u})
$$

As in Maragos and Bovik (1995); Maragos et al. (1993a), let us consider a band limited envelope $A(\mathbf{u})$, i.e $\operatorname{FT}[A(\mathbf{u})]=\tilde{A}(\boldsymbol{\omega})=0$ for $\|\boldsymbol{\omega}\| \geq \omega_{A}$ where FT[] is the Fourier Transform. Giving a mean spectral absolute value:

$$
\mu_{A}=\frac{1}{(2 \pi)^{n}} \int_{-\omega_{A}}^{+\omega_{A}} \ldots \int_{-\omega_{A}}^{+\omega_{A}}|\tilde{A}(\boldsymbol{\omega})| d \omega_{1} d \omega_{2} \ldots d \omega_{n}
$$

we deduce the following relations for any partial derivatives giving $r=r_{1}+r_{2}+\cdots+r_{n}$ :

$$
\begin{aligned}
& \mathrm{FT}\left[\frac{\partial^{r} A}{\partial x_{1}^{r_{1}} \partial x_{2}^{r_{2}} \ldots \partial x_{n}^{r_{n}}}\right]=\left(j \omega_{1}\right)^{r_{1}}\left(j \omega_{2}\right)^{r_{2}} \ldots\left(j \omega_{n}\right)^{r_{n}} \tilde{A}(\boldsymbol{\omega}) \\
& \Rightarrow\left|\frac{\partial^{r} A}{\partial x_{1}^{r_{1}} \partial x_{2}^{r_{2}} \ldots \partial x_{n}^{r_{n}}}\right|=\left|\mathrm{FT}^{-1}\left[\left(j \omega_{1}\right)^{r_{1}}\left(j \omega_{2}\right)^{r_{2}} \ldots\left(j \omega_{n}\right)^{r_{n}} \tilde{A}(\boldsymbol{\omega})\right]\right| \leq \omega_{A}^{r_{A}} \mu_{A}
\end{aligned}
$$

Further, the quantity $\frac{\partial^{r} A}{\partial x_{1}^{r_{1}} \partial x_{2}^{r_{2}} \ldots \partial x_{n}^{r_{n}}}$ being the general term of the higher order gradient $\frac{d^{r} A}{d \mathbf{u}^{r}}$, it follows from (56), and the results $(52,53)$ obtained in Appendix B linking the higher order gradients and directional derivatives that:

$$
\begin{aligned}
& \text { for an even order } r=2 p \text { : } \\
& \left|\frac{\partial^{r} A}{\partial \mathbf{v}^{r}}(\mathbf{u})\right| \leq n^{r} \omega_{A}^{r} \mu_{A}\left\|\bigotimes_{p} \mathbf{v}^{T}\right\| \cdot\left\|\bigotimes_{p} \mathbf{v}\right\| \\
& \text { for an odd order } r=2 p+1: \\
& \left|\frac{\partial^{r} A}{\partial \mathbf{v}^{r}}(\mathbf{u})\right| \leq n^{r} \omega_{A}^{r} \mu_{A}\left\|\bigotimes_{p} \mathbf{v}^{T}\right\| \cdot\left\|\bigotimes_{(p+1)} \mathbf{v}\right\|
\end{aligned}
$$

Moreover, the following result is deduced from the properties of the Kronecker product, provided that the dimensions are compatible:

$$
\left(A_{1} \otimes A_{2} \otimes \cdots \otimes A_{n}\right)\left(B_{1} \otimes B_{2} \otimes \cdots \otimes B_{n}\right)=\left(A_{1} B_{1}\right) \otimes\left(A_{2} B_{2}\right) \otimes \cdots \otimes\left(A_{n} B_{n}\right)
$$

Equation (58) yields:

$$
\begin{aligned}
& \left\|\bigotimes_{p} \mathbf{v}\right\|^{2}=\bigotimes_{p} \mathbf{v}\left(\bigotimes_{p} \mathbf{v}\right)^{T}=\bigotimes_{p} \mathbf{v} \bigotimes_{p} \mathbf{v}^{T} \\
& =\left(\mathbf{v} \mathbf{v}^{\mathbf{T}}\right) \otimes\left(\mathbf{v} \mathbf{v}^{\mathbf{T}}\right) \otimes \cdots \otimes\left(\mathbf{v} \mathbf{v}^{\mathbf{T}}\right) \\
& =\|\mathbf{v}\|^{2} \otimes\|\mathbf{v}\|^{2} \otimes \cdots \otimes\|\mathbf{v}\|^{2} \\
& =\|\mathbf{v}\|^{2 p}
\end{aligned}
$$

Finally:

$$
\left\|\bigotimes_{p} \mathbf{v}\right\|=\|\mathbf{v}\|^{p}
$$


Thus Eqs. $(56,57)$ and $(60)$ yield for any order $r$ :

$$
\left|\frac{\partial^{r} A}{\partial \mathbf{v}^{r}}(\mathbf{u})\right| \leq n^{r} \mu_{A} \omega_{A}^{r}\|\mathbf{v}\|^{r}
$$

Let us express now the first and second order directional derivatives of $A(\mathbf{u})$ according to a normalized vector $\|\mathbf{v}\|=1$ :

$$
\left|\frac{\partial A}{\partial \mathbf{v}}(\mathbf{u})\right| \leq n \omega_{A} \mu_{A} \quad\left|\frac{\partial^{2} A}{\partial \mathbf{v}^{2}}(\mathbf{u})\right| \leq n^{2} \omega_{A}^{2} \mu_{A}
$$

Finally, we deduce the following inequality:

$$
\left|\Phi_{2, \mathbf{v}}[A(u)]\right| \leq n^{2} \omega_{A}^{2} \mu_{A}^{2}+n^{2} \omega_{A}^{2} \mu_{A} A_{\max }
$$

For narrow band signals analysis, we express each local component frequency as a sum of a constant carrier term and an excursion frequency:

$$
w_{i}(\mathbf{u})=w_{c, i}+w_{m, i} f_{i}(\mathbf{u})
$$

As in Maragos and Bovik (1995), we assume that $f_{i}(\mathbf{u}) \in[-1,+1]$ and $w_{m, i}$ is the maximum deviation of the frequency from its center value $0 \leq w_{m, i} \ll\left|w_{c, i}\right|$. Let us now compute the higher order derivatives of $B(\mathbf{u})=\cos (\phi(\mathbf{u}))$ according to the vector $\mathbf{v}$

$$
\begin{aligned}
\frac{\partial B}{\partial \mathbf{v}} & =\frac{\partial \phi}{\partial \mathbf{v}} \sin (\phi(\mathbf{u})) \\
\frac{\partial^{2} B}{\partial \mathbf{v}^{2}} & =\frac{\partial^{2} \phi}{\partial \mathbf{v}^{2}} \sin (\phi(\mathbf{u}))-\left(\frac{\partial \phi}{\partial \mathbf{v}}\right)^{2} \cos (\phi(\mathbf{u}))
\end{aligned}
$$

We suppose that the excursion $\Delta_{i} j=w_{m, i} f_{i}(\mathbf{u})$, for all $i$ is such that $f_{i}(\mathbf{u})$, is a band limited signal i.e, $\operatorname{FT}\left[f_{i}(\mathbf{u})\right]=\tilde{f}_{i}(\boldsymbol{\omega})=0$ for $\|\boldsymbol{\omega}\| \geq \omega_{f_{i}}$. We suppose that $\omega_{f_{i}} \leq \omega_{c_{i}}$ where $\omega_{c}=\left\|\mathbf{w}_{\mathbf{c}}(\mathbf{u})\right\|$ corresponds to the norm of the local central frequency, which means that the frequency excursion is not too fast. The mean spectral absolute value is given by:

$$
\mu_{f_{i}}=\frac{1}{(2 \pi)^{n}} \int_{-\omega_{f_{i}}}^{+\omega_{f_{i}}} \ldots \int_{-\omega_{f_{i}}}^{+\omega_{f_{i}}}\left|\tilde{f}_{i}(\boldsymbol{\omega})\right| d \omega_{1} d \omega_{2} \ldots d \omega_{n}
$$

We compute the second order derivative along a normalized vector $\mathbf{v}=\left(v_{1}, \ldots, v_{n}\right)^{T}$ :

$$
\frac{\partial^{2} \phi}{\partial \mathbf{v}^{2}}(\mathbf{u})=\mathbf{v}^{T} \frac{d^{2} \Phi}{d \mathbf{u}^{2}} \mathbf{v}=\sum_{i=1}^{n} \sum_{j=1}^{n} v_{i} w_{m_{i}} \frac{\partial f_{i}}{\partial x_{j}} v_{j}
$$

A similar inequality as Eq. (56) and the previous conditions lead to:

$$
\left|\frac{\partial^{2} \phi}{\partial \mathbf{v}^{2}}(\mathbf{u})\right| \ll \sum_{i=1}^{n} \sum_{j=1}^{n} w_{c_{i}}^{2} \mu_{f_{i}}
$$

The choice of $\mu_{f_{i}} \simeq 1$ means that the FT of the functions $f_{i}$ own linear phases (Maragos et al. 1993a). Then, the inequality (67) becomes:

$$
\left|\frac{\partial^{2} \phi}{\partial \mathbf{v}^{2}}(\mathbf{u})\right| \ll \sum_{i=1}^{n} \sum_{j=1}^{n} w_{c_{i}}^{2}=n \omega_{c}^{2}
$$


On the other hand, an appropriate choice of the vector $\mathbf{v}$ leads to:

$$
\frac{\partial \phi}{\partial \mathbf{v}}(\mathbf{u})=\frac{d \Phi}{d \mathbf{u}} \mathbf{v} \geq\|\mathbf{w}(\mathbf{u})\| \simeq w_{c}
$$

For example the following vector used in our study, satisfies (69):

$$
\mathbf{v}=\left(\frac{\operatorname{sign}\left(w_{1}\right)}{\sqrt{n}}, \ldots, \frac{\operatorname{sign}\left(w_{n}\right)}{\sqrt{n}}\right)^{T}
$$

Finally, it is trivial to generalize the well known property of the application of the TKEO to the product of functions, regarding the directional derivatives:

$$
\begin{aligned}
\Phi_{2, \mathbf{v}}[A(\mathbf{u}) B(\mathbf{u})] & =A^{2}(\mathbf{u}) \Phi_{2, \mathbf{v}}[B(\mathbf{u})]+B^{2}(\mathbf{u}) \Phi_{2, \mathbf{v}}[A(\mathbf{u})] \\
& =A^{2}(\mathbf{u})\left(\left(\frac{\partial \phi}{\partial \mathbf{v}}\right)^{2}-\frac{1}{2} \frac{\partial^{2} \phi}{\partial \mathbf{v}^{2}} \sin 2 \phi(\mathbf{u})\right)+\Phi_{2, \mathbf{v}}[A(\mathbf{u})] \cos \phi(\mathbf{u})
\end{aligned}
$$

According to our hypothesis, the instantaneous envelope $A(\mathbf{u})$ is a slow signal, relatively to the carrier one, which implies that $\omega_{A} \ll \omega_{c}$. Moreover, the envelope being observed on a short time interval, it is possible to extrapolate it as a sinusoidal function i.e, $\mu_{A} \simeq A_{\max }$. Then the equality (70) yields:

$$
\Phi_{2, \mathbf{v}}[A(\mathbf{u}) B(\mathbf{u})]=A^{2}(\mathbf{u})\left(\frac{\partial \phi}{\partial \mathbf{v}}\right)^{2}+\varepsilon_{\mathbf{v}}(\mathbf{u})
$$

According to Eqs. (63) and (68) and the previous assumptions, the error term $\varepsilon_{\mathbf{v}}$ verifies:

$$
\left|\varepsilon_{\mathbf{v}}(\mathbf{u})\right| \ll A_{\max }^{2}\left(\frac{1}{2} n \omega_{c}^{2}+2 n^{2} \omega_{c}^{2}\right)
$$

When comparing to error bound detailed in Maragos and Bovik (1995), there is a multiplicative factor $n$. In general, for any vector $\mathbf{v}$ the operator leads to:

$$
\begin{aligned}
\Phi_{2, \mathbf{v}}[A(\mathbf{u}) \cos \phi(\mathbf{u})]= & A^{2}(\mathbf{u})\left(\left(\frac{\partial \phi}{\partial \mathbf{v}}\right)^{2}-\mathbf{v}^{T} \frac{1}{2} \frac{d^{2} \phi}{d \mathbf{u}^{2}} \sin 2 \phi(\mathbf{u}) \mathbf{v}\right) \\
& +\mathbf{v}^{T} \Psi_{2, \mathbf{v}}[A(\mathbf{u})] \cos \phi(\mathbf{u}) \mathbf{v}
\end{aligned}
$$

Notice tha it is possible to find a vector that minimizes the error term and yields an energy operator which output is proportional to the quantity $A(\mathbf{u})\|w(\mathbf{u})\|^{2}$. This corresponds to a classical constraint minimization problem i.e:

$$
\begin{aligned}
& \operatorname{minimize} \mathbf{v}^{T} \mathbf{R v} \text { subject to } \mathbf{v}^{T} \mathbf{w}=\|\mathbf{w}\| \\
& \text { where : } \mathbf{R}=\frac{1}{2} \frac{d^{2} \phi}{d \mathbf{u}^{2}}+\Psi_{2, \mathbf{v}}[A(\mathbf{u})]
\end{aligned}
$$

Provided that the matrix $\mathbf{R}$ is not a singular one, a solution to this problem is given by:

$$
\mathbf{v}=\frac{\mathbf{R}^{-1} \mathbf{w}}{\mathbf{w}^{T} \mathbf{R}^{-1} \mathbf{w}}\|\mathbf{w}\|
$$

Considering a slow varying envelope in a short time interval, the optimal vector depends on the Hessian of the phase and the frequency. 


\section{References}

Boudraa, A., \& Diop, E. (2008). Image contrast enhancement based on 2D Teager-Kaiser operator. In IEEE international conference on image processing, San Diego, USA, pp. 3180-3183.

Boudraa, A., Salzenstein, F., \& Cexus, J. (2005). Two-dimensional continuous higher-order energy operators. Optical Engineering, 44(11), 7001-7010.

Boudraa, A., Bouchikhi, A., \& Diop, E. (2008). Teager-Kaiser energy bi-level thresholding. In IEEE international symposium on communications, control and signal processing, Malta, pp. 1086-1090.

Bovik, A., Gopal, N., Emmoth, T., \& Restrepo, A. (1992). Localized measurement of emergent image frequencies by Gabor wavelets. IEEE Transactions on Information Theory, 38(2), 691-712.

Brewer, J. (1978). Kronecker products and matrix calculus in system theory. IEEE Transactions Circuits and Systems, 25(9), 772-781.

Cai, Q. W., Wei, P., \& Xiao, X. (2010). Single-channel blind separation of overlapped multicomponents based on energy operator. Science China Information Sciences, 53(1), 147-157.

Dimitriadis, D., Potamianos, A., \& Maragos, P. (2009). A comparison of the squared energy and Teager-Kaiser operators for short-term energy estimation in additive noise. IEEE Transactions Signal Processing, 57(7), 2569-2581.

Felsberg, M., \& Granlund, G. (2004). POI detection using channel clustering and the 2D energy tensor. In Pattern recognition: 26th DAGM symposium, LNCS, Vol. 3175, (pp. 103-110). Berlin: Springer.

Felsberg, M., \& Jonsson, E. (2005). Energy tensors: Quadratic phase invariant image operators. In DAGM 2005, LNCS, Vol. 3663, (pp. 493-500). Springer.

Felsberg, M., \& Köthe, U. (2005). Get: The connection between monogenic scale-space and gaussian derivatives. In R. Kimmel, N. Sochen, \& J. Weickert (Eds.), Scale space and PDE methods in computer vision, LNCS, Vol. 3459 (pp. 192-203). Springer.

Havlicek, J., Tay, P., \& Bovik, A. (2005). Handbook of image and video processing, 2nd edn, chap AM-FM image models: Fundamental techniques and emerging trends (pp. 377-395). Burlington: Elsevier Academic Press.

Kaiser, J. (1990). On a simple algorithm to calculate the energy of a signal. In Proceedings of ICASSP, pp. 381-384.

Kokkinos, I., Evangelopoulos, G., \& Maragos, P. (2009). Texture analysis and segmentation using modulations features, generative models and weighted curve evolution. IEEE Transactions Pattern Analysis Machine Intelligent, 31(1), 142-157.

Larkin, K. (1996). Efficient nonlinear algorithm for envelope detection in white light interferometry. Journal of Optical Society of America A, 13, 832-843.

Larkin, K. (2005). Uniform estimation of orientation using local and nonlocal 2-D energy operators. Optics Express, 13(20), 8097-8121.

Maragos, P., \& Bovik, A. (1995). Image demodulation using multidimensional energy separation. Journal of Optical Society of America A, 12, 1867-1876.

Maragos, P., \& Potamianos, A. (1995). Higher order differential energy operators. IEEE Signal Processing Letters, 2, 152-154.

Maragos, P., Kaiser, J., \& Quatieri, T. (1993). On amplitude and frequency demodulation using energy operators. IEEE Transactions Signal Processing, 41(4), 1532-1550.

Maragos, P., Quatieri, T., \& Kaiser, J. (1993). Energy separaton in signal modulations with applications to speech analysis. IEEE Transactions Signal Processing, 41, 3024-3051.

Mitra, S., Li, H., Lin, I., \& Yu, T. (1991). A new class of nonlinear filters for image enhancement. In Proceedings of ICASSP, Vol. 91, pp. 2525-2528.

Moon, T. K., \& Stirling, W. C. (2000). Mathematical methods and algorithms for signal processing. Upper Saddle River, NJ: Prentice Hall.

Ramponi, G., Strobel, N., Mitra, S., \& Yu, T. (1996). Nonlinear unsharp masking methods for image contrast enhancement. Journal of Electronic Imaging, 5(3), 353-366.

Salzenstein, F., \& Boudraa, A. (2009). Multi-dimensional higher order differential operators derived from the Teager-Kaiser energy tracking function. Signal Processing, 89(4), 623-640.

Salzenstein, F., Montgomery, P., Montaner, D., \& Boudraa, A. (2005). Teager-Kaiser energy and higher order operators in white light interference microscopy for surface shape measurement. EURASIP Journal of Applications on Signal Processing, 17, 2804-2815.

Salzenstein, F., Boudraa, A., \& Cexus, J. (2007). Generalized higher-order nonlinear energy operators. Journal of Optical Society of America A, 24, 3717-3727.

Vakman, D. (1996). On the analytic signal, the Teager-Kaiser energy algorithm, and other methods for defining amplitude and frequency. IEEE Transactions on Signal Processing, 44(4), 791-797.

Vetter, W. J. (1973). Matrix calculus operations and Taylor expansions. SIAM Review, 15(2), 352-369. 
Vleesschauwer, D. D., Cheikh, F. A., Hamila, R., \& Gabbouj, M. (1997). Watershed segmentation of image enhanced by Teager energy driven diffusion. In Proceedings of sixth conference image processing and its applications, pp 254-258.

Yu, T., Mitra, S., \& Kaiser, J. (1991). A novel nonlinear filter for image enhancement. In Proceedings of SPIE/SPSE symposium on electronic imaging: Science and technology, pp. 303-309.

\section{Author Biographies}
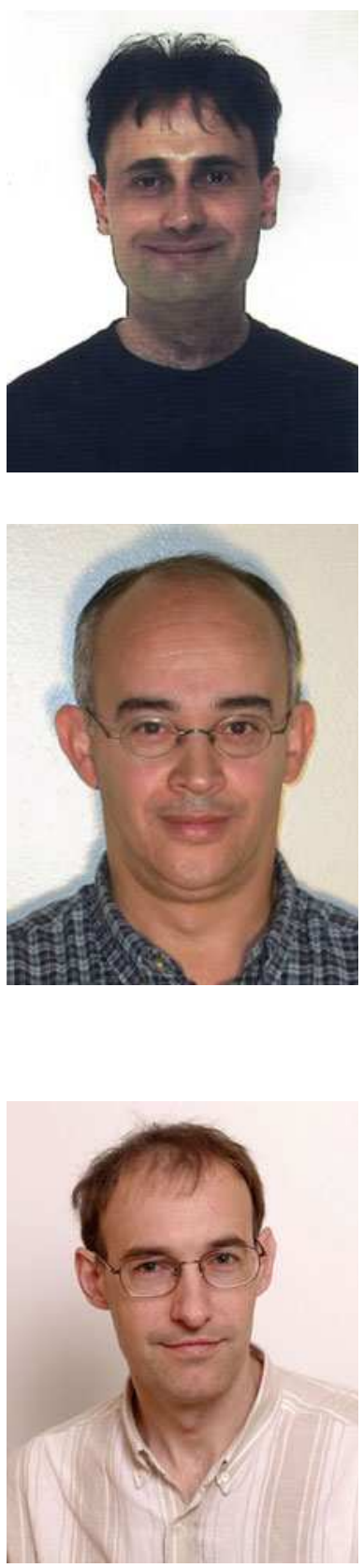

F. Salzenstein was born in Nice, France. He received the Engineering degree from. l'Ecole Nationale Supérieure des Télécommunications de Bretagne, Brest, France. He received the Ph.D. degree in Image Processing from the University of Rennes 1, in collaboration with Institut National des Telecommunications, Evry, France. He worked as Engineer in the Air Trafic control, Thomson CSF, France. $\mathrm{He}$ is currently Associate Professor of Electrical Engineering at Université de Strasbourg, France. His current research interests include non linear operators, data fusion, time frequency analysis, Markovian methods, fuzzy computer vision applied to the Astronomy.

A. O. Boudraa was born in Constantine, Algeria. He received the B.S. degree in Physics (Electronics Engineering) from Constantine Institute of Physics, University of Constantine, Algeria, and the Engineer degree in Electronics from Educatel (Liege, Belgium). He also received the M.S. degree in Biomedical Engineering from INSA, Lyon, University degree in Nuclear Magnetic Resonance, the Ph.D. degree in Image Processing and the University degrees in Statistics and Modeling and in Positron Emission Tomography all from the University of Claude Bernard, Lyon 1, France. He is currently Associate Professor of Electrical Engineering at Ecole Navale, Brest, France. His current research interests include computer vision, vector quantization, data structures and analysis, data fusion, time frequency analysis, higher-order energy operators, empirical mode decomposition, hard and fuzzy pattern recognition. Dr. Boudraa is recipient of 2003 Varian Prize awarded by the Swiss Society of Radiobiology and Medical Physics for the best published paper impacting Radiation Oncology. Dr. BOUDRAA is a Senior Member IEEE.

T. Chonavel received the Ph.D. thesis in signal processing from ENST, Paris France, in 1992. His thesis was about trigonometric moment problems and array processing. He joined Institut Telecom (Institut Telecom) in 1993, where he is currently Professor at telecom Bretagne, Brest, France. His researches are about signal processing and communications with works in adaptive processing, Radar, and applications related to underwater acoustics (ocean tomography and seismic). 